Working Paper 03-29

Economics Series 08

May 2003
Departamento de Economía

Universidad Carlos III de Madrid

Calle Madrid, 126

28903 Getafe (Spain)

Fax (34) 916249875

\title{
A HERDING PERSPECTIVE ON GLOBAL GAMES AND MULTIPLICITY *
}

\author{
James S. Costain ${ }^{1}$
}

\begin{abstract}
Recently, it has been claimed that full-information multiple equilibria in games with strategic complementarities are not robust, because generalizing to allow slightly heterogeneous information implies uniqueness. This paper argues that this "global games" uniqueness result is itself not robust. If we generalize by allowing most agents to observe a few previous actions before choosing, instead of forcing players to move exactly simultaneously, then multiplicity of outcomes is restored. Only a small sample of observations is needed to make our herding equilibrium behave like a full-information sunspot equilibrium instead of a global games equilibrium.
\end{abstract}

Keywords: Herding, information cascades, multiplicity, uniqueness, heterogeneity, strategic complementarities, global games, adaptive learning, stochastic recursive algorithms

JEL Classification: C62, C72, C73, E00

${ }^{1}$ Departamento de Economía, Universidad Carlos III de Madrid. E.mail: jcostain@eco.uc3m.es

\footnotetext{
* Thanks to Antonio Ciccone for encouraging me to study some related questions. Thanks also for helpful comments to Fabrizio Germano, Joel Shapiro, Stephen Morris, Hyun Song Shin, Frank Heinemann, Dan Friedman, Berthold Herrendorf, Marcel Jansen, Juan Ruiz, and Antonio Cabrales. This paper was first presented under the title "On Multiplicity and Heterogeneity in a Herding Model", at CEMFI, where I thank Guillermo Caruana and other seminar participants. Financial support from the Spanish Ministry of Science and Technology (MCyT grant SEC2002-01601) is gratefully acknowledged. Errors are the responsibility of the author.
} 


\section{Introduction}

Must two economies with the same aggregate fundamentals experience the same aggregate outcome? This question is not new, but it remains controversial. If macroeconomic outcomes are not always uniquely determined by fundamentals, there may be important implications for policy making: on one hand, selecting the preferred outcome might be part of the role of policy; but on the other hand, activist policy is itself sometimes cited as a source of nonuniqueness (e.g. Schmitt-Grohe and Uribe 2001).

Different areas of literature have formalized the question of uniqueness of aggregate outcomes in different ways. One way in which the outcome associated with a given fundamental might fail to be unique is that the structure of the economy might permit multiple equilibria. But even within a single equilibrium, some classes of models with a network structure allow tiny shocks at the micro level to snowball into different aggregate outcomes, as occurs in the literature on "herding".

Both multiple equilibrium models and herding models have been criticized recently, on the grounds that existing examples of multiplicity may not be robust. This paper evaluates some of these recent criticisms by comparing three games which have the same payoff functions but different information sets: a full-information game, a global game, and a herding game. The main finding is that multiplicity of aggregate outcomes is indeed robust, especially if strategic complementarities are strong.

\subsection{The global games literature}

Ever since Cooper and John (1988), it has been widely known that multiple equilibria may arise in models with strategic complementarities. But multiplicity has often been studied in the context of simultaneous-move, representativeagent games. Various authors have suggested recently that multiplicity might be fragile in the face of heterogeneous agents. Herrendorf, Valentinyi, and Waldmann (2000) demonstrate that sufficient payoff heterogeneity eventually 
eliminates multiplicity by driving all agents towards corner solutions, assuming that the choice set is bounded. ${ }^{1}$

A more dramatic sort of fragility arises in "global games", as defined by Carlsson and van Damme (1993). In this context, though a simultaneous-move, representative-agent game may allow multiple equilibria, the introduction of an infinitesimal amount of heterogeneity in agents' information about aggregate fundamentals often suffices to eliminate the multiplicity, leading to a unique aggregate outcome for each aggregate state (Morris and Shin 1998, 2002). ${ }^{2}$ That is, equilibrium is unique as we approach the limit of full information, in spite of the fact that the full information game itself exhibits multiplicity.

This theoretical result, usually proved via interated dominance arguments, holds for a surprisingly large class of games (Frankel, Morris, and Pauzner, 2003), and is also supported by experimental evidence. Cabrales, Nagel, and Armenter (2002) and Heinemann, Nagel, and Ockenfels (2002) ask subjects to play one-shot, simultaneous-move, binary-choice games that have multiple equilibria under full information. As the global games literature would claim, their results show a unique mapping from aggregate fundamentals to the aggregate proportions making each choice.

While this evidence is consistent with the global games literature, it also raises an obvious question: would results differ if players chose in sequence rather than moving simultaneously? After all, if we regard each treatment as a random draw from the same population of potential players, it is extremely hard to see how different treatments could lead to significantly different aggregate outcomes if any given game is played only once (so that there is no way to learn) and if players move simultaneously (so that they cannot condition on other players' behavior). Allowing sequential (or repeated) play might allow aggregate behavior to evolve over time, possibly reaching different outcomes even in the same state of aggregate fundamentals.

\footnotetext{
${ }^{1}$ See Ciccone and Costain (2001) for discussion.

${ }^{2}$ Often, though, increasing heterogeneity further brings back multiplicity.
} 


\subsection{The herding literature}

The idea that sequential play might give rise to multiple aggregate outcomes under the same aggregate fundamentals motivates the literature on "herding" or "information cascades" (Banerjee 1992; Bikhchandani, Hirshleifer, and Welch, 1992, 1996, 1998). In these models, agents want to choose an action consistent with an aggregate fundamental, but this fundamental is unknown. Each observes a private signal correlated with the fundamental, and they choose in sequence, taking into account the actions of those who chose before them. Multiple outcomes are possible because players may rationally follow the actions of others, ignoring their own signals, if the information inferred from previous actions outweighs that derived from their own signals. In other words, multiple outcomes can occur because aggregation of the information in the signals fails.

However, there is also controversy about the robustness of herding behavior. Initial examples, involving a signal with only two possible values, showed that an infinite sequence of players could choose the "wrong" action (the one that is Pareto dominated under the true aggregate state). But later work showed that average play always converges to the "right" action if the signal has continuous support (Vives 1993). Chamley (2002), responding to Smith and Sorenson (2002), confirms this result, but argues that the convergence to the unique aggregate outcome is so slow that it may be uninteresting in practice. Chari and Kehoe (2002) argue that multiple outcomes of herding are robust if the timing of decisions is endogenous.

\subsection{Contribution of this paper}

From the perspective of the global games literature, this paper can be seen as another robustness exercise. Does the uniqueness result that arises near the full-information limit of a global game still obtain, if we assume sequential rather than simultaneous choice?

Interestingly, in the herding model that we study, there are two ways to take the full-information limit. If we approach full information by making each 
idiosyncratic signal arbitrarily accurate, then we find a partial confirmation of the global games uniqueness result. However, if we approach full information by increasing the average number of previous choices observed, so that most players can infer the average choice of others, then multiple outcomes are preserved, and aggregate behavior resembles a sunspot equilibrium.

From the perspective of the herding literature, one contribution of this paper is a more realistic, less delicate, more easily generalizable information structure that generates herding behavior. Rather than assuming a fixed, known sequence in which everyone conditions on all previous choices, we impose a Markovian structure in which each player observes a random number of previous choices. This simplifies some of the analysis, and more importantly, it also allows us to study how the equilibrium changes when we vary the amount of information obtained by observing others.

As in Chamley (2002), our model sometimes leads not to multiplicity but instead to extremely slow convergence - especially when information aggregation problems are more important than strategic complementarities. But it is unclear why the herding literature has focused so much on information revelation, when many of the economic contexts to which herding models have been applied (currency crises, bank runs, ...) seem to call for a model with strategic complementarities. We show that stronger strategic complementarities increase the range of parameters and aggregate states over which multiplicity occurs, regardless of whether the signal has binary or continuous support.

\section{The herding model}

Like much of the global games literature, we consider a one-shot game; but in contrast, we assume that choice is not exactly simultaneous. Players choose between two actions, 0 and 1, which we sometimes call "bond" and "stock", respectively. The payoff to "stock" is greater if the fraction of the population playing stock, $S$, is greater; but it also depends on the aggregate state $Z$. Neither $S$ nor $Z$ is known. An individual's assessment of $Z$ and $S$ is inferred from a signal $\sigma$ correlated with $Z$, and also by observing some previous choices. 
The first information cascade models, of Banerjee (1992) and Bikhchandani, Hirshleifer, and Welch (1992), assume that individuals make their decisions in a known, fixed order, and observe all preceding choices. We assume instead that individuals do not know their position in the sequence of choices. Players observe a random number $n$ of previous choices - less than or equal to some finite $N^{m a x}$ — but they have no other information about their position in the sequence. The choices they observe are selected with equal probability from all those who have gone before.

This specification cleans up the analysis in several ways. First, unlike the case where each player conditions on a history of a different length, here each has the same strategy space, choosing an action that depends on $n$, on the number of times $m$ that they observe "stock", and on their signal $\sigma$. Second, with extremely limited information about their positions in the infinite sequence, players conclude that the probability of being near the beginning of the sequence is negligible. Third, since all predecessors are equally likely to be observed, each observation can be treated as an independent random draw from the whole population, in which the fraction playing stock is $S$. Therefore, the key to the analysis is to calculate the equilibrium value(s) of $S$.

\subsection{The individual's problem}

Individuals $i$ in a countably infinite sequence $\mathcal{I} \equiv\{1,2,3, \ldots\}$ each choose an action $x_{i} \in\{0,1\}$. They choose in order: person $i=1$ goes first, person 2 second, and so forth. The notation $\mathcal{I}_{i} \equiv\{1,2, \ldots i\}$ refers to the first $i$ agents.

All players have the same utility function $u(x, Z, S, \sigma)$. Here $Z \in \mathcal{Z}$ is the random aggregate state (the fundamental) of the economy; we call a higher $Z$ "good" and a lower $Z$ "bad". The cumulative probability distribution of $Z$ is $\zeta(Z) . S$ is the fraction of the population choosing to play "stock"; that is,

$$
S \equiv \lim _{I \rightarrow \infty} \frac{1}{I} \sum_{i=1}^{I} x_{i} \in[0,1]
$$

The pair $(Z, S)$ of exogenous and endogenous aggregate variables that enter the utility function will be called the aggregate outcome. The space of possible 
outcomes is $\Omega \equiv \mathcal{Z} \times[0,1]$. Individuals cannot directly observe the true state of the economy $Z$ or the aggregate choice $S$. Instead, they must try to infer the aggregate situation on the basis of a limited information set, and maximize expected utility accordingly. Each person observes a signal $\sigma_{i} \in \mathcal{R}$ which is correlated with the aggregate state. Conditional on $Z$, it is independently and identically distributed across individuals, with c.d.f. $F(\sigma \mid Z)$. If $Z_{H}>Z_{L}$, then the distribution of $\sigma$ conditional on $Z=Z_{H}$ first-order stochastically dominates the distribution when $Z=Z_{L}$, so that a high $\sigma_{i}$ indicates a good aggregate state. We assume that $F$ is continuous and has unbounded support; we call its density $f(\sigma \mid Z)$.

We consider a utility function that nests three special cases related to important factors from the literatures on herding and multiplicity: information revelation, strategic complementarities, and payoff heterogeneity. The first special case is that of pure information revelation:

$$
u^{\text {info }}(x, Z)=x Z+(1-x)(1-Z)
$$

With these preferences, the player prefers stock if and only if $Z \geq 1 / 2$. The case of strategic complementarities only is given by:

$$
u^{s c}(x, S)=x S+(1-x)(1-S)
$$

which implies that stock is preferred whenever $S \geq 1 / 2$. The third special case is payoff heterogeneity only:

$$
u^{\text {het }}(x, \sigma)=x \sigma-(1-x) \sigma
$$

so that stock is preferred whenever $\sigma$ is positive. Our general utility function is a weighted average of all three:

$$
u(x, Z, S, \sigma)=w^{h e t} u^{h e t}(x, \sigma)+w^{s c} u^{s c}(x, S)+w^{\text {info }} u^{\text {info }}(x, Z)
$$

where without loss of generality we set $w^{\text {info }}=1-w^{s c}-w^{\text {het }}$.

In order to generate herding behavior, we must give players some information about others' choices, beyond that inferred from the private signals. Here, 
we suppose that people observe a random number of previous choices. For simplicity, we assume that person $i$ 's observations are drawn with equal probability (but without replacement) from her set of predecessors $\mathcal{I}_{i-1} \equiv\{1,2, \ldots, i-1\}$, so that $i$ is just as likely to observe the choice of agent 1 as that of agent $i-1$ or any other preceding player. We assume that $i$ can observe up to

$$
N_{i}^{\max } \equiv \min \left(N^{\max }, i-1\right)
$$

predecessors, each with independent probability $q$. Thus the number of previous actions $n$ observed by $i$ has the following binomial distribution:

$$
N_{i}(n) \equiv \operatorname{prob}\left(n_{i}=n \mid i\right)=\left(\begin{array}{c}
N_{i}^{\max } \\
n
\end{array}\right) q^{n}(1-q)^{N_{i}^{\max }-n}
$$

For $i>N^{\max }$, this distribution does not depend on $i$, so we can write it as

$$
N(n) \equiv\left(\begin{array}{c}
N^{\max } \\
n
\end{array}\right) q^{n}(1-q)^{N^{\max }-n}
$$

The number of observed predecessors who play "stock" is denoted $m_{i}$.

Finally, we make the crucial assumption that individuals do not know their position in the sequence. That is, player $i$ does not know that she is the $i$ th person to choose. She knows only that her position cannot be less than $n_{i}+1$, since she has observed $n_{i}$ previous choices. Thus, her information set is:

$$
\epsilon_{i} \equiv\left(n_{i}, m_{i}, \sigma_{i}\right)
$$

The set of possible individual states is $\mathcal{E} \equiv\left\{0,1, \ldots N^{\max }\right\} \times\left\{0,1, \ldots N^{\max }\right\} \times \mathcal{R}$. Given her information $\epsilon \in \mathcal{E}$, the player must try to deduce the aggregate situation $(Z, S) \in \Omega$; we denote her conditional probability assessment as:

$$
\Pi(z, s \mid n, m, \sigma) \equiv \operatorname{prob}(Z \leq z, S \leq s \mid n, m, \sigma)
$$

In general, the individual can choose a probability of playing $x_{i}=1$ conditional on any idiosyncratic state $\epsilon$. We denote this probability as $y(n, m, \sigma)$, which is a function mapping $\mathcal{E}$ into $[0,1]$. Thus, the expected utility maximization problem can be defined pointwise in $\mathcal{E}$ as: 
Individual's problem:

$$
y(n, m, \sigma) \in \underset{y \in[0,1]}{\operatorname{argmax}} \int_{(Z, S) \in \Omega}[y u(1, Z, S, \sigma)+(1-y) u(0, Z, S, \sigma)] d \Pi(Z, S \mid n, m, \sigma)
$$

Actually, allowing for mixed strategies is more general than necessary. Generically (in terms of possible distributions $\Pi$ ) a player will strictly prefer 0 or 1 for almost every $\epsilon$. Thus policy can normally be described by threshold functions $\theta(n, m)$ representing the signals at which players are indifferent between stock and bond. Since a higher $\sigma$ indicates higher $Z$, and a higher payoff to stock, we will search for threshold strategies of the following form:

$$
\begin{array}{ll}
y(n, m, \sigma)=1 & \text { iff } \quad \sigma \geq \theta(n, m) \\
y(n, m, \sigma)=0 & \text { iff } \quad \sigma<\theta(n, m)
\end{array}
$$

but we will also check whether this is in fact optimal in our simulations.

\section{$2.2 \quad$ Equilibrium}

Any policy function $y(n, m, \sigma)$ induces a stochastic process $x_{i}$ for $i \in \mathcal{I}$. From this stochastic process we will also define

$$
X_{i} \equiv \sum_{j=1}^{i} x_{i} \quad \text { and } \quad S_{i} \equiv \frac{1}{i} X_{i} \in \mathcal{J}_{i} \equiv\left\{0, \frac{1}{i}, \frac{2}{i}, \ldots 1\right\}
$$

so that we have $S=\lim _{i \rightarrow \infty} S_{i}$. Note that $S_{i}$ can also be calculated recursively:

$$
S_{i}=S_{i-1}+\frac{1}{i}\left(x_{i}-S_{i-1}\right)
$$

This representation is useful because it shows that each history $\left\{S_{i}\right\}_{i=1}^{\infty}$ has the structure of a stochastic recursive algorithm, which suggests that we can use results from the adaptive learning literature to analyze convergence of $S_{i}$.

To spell out the stochastic processes for $x_{i}$ and $S_{i}$, recall first that the c.d.f. of the aggregate fundamental is $\zeta(Z)$. Next, for each $i \in \mathcal{I}$, a signal $\sigma_{i}$ is drawn with distribution $F(\sigma \mid Z)$. The number of observations $n_{i}$ is drawn with distribution $N_{i}(n)$. Then if the fraction of predecessors who have played stock 
is $S_{i}=s$, (so that the number who have played stock is $X_{i}=i s$ ) the probability that exactly $m$ predecessors played stock, out of $n_{i}=n$ observations, is

$$
M_{i}(m \mid n, s) \equiv \operatorname{prob}\left(m_{i}=m \mid i, n, s\right) \equiv\left(\begin{array}{c}
n \\
m
\end{array}\right)\left(\frac{1}{i !}\right)\left(\frac{(s i) !}{(s i-m) !}\right)\left(\frac{(i-s i) !}{(i-s i+m-n) !}\right)
$$

which approaches the following binomial limiting distribution if $i$ is large:

$$
M(m \mid n, s) \equiv\left(\begin{array}{c}
n \\
m
\end{array}\right) s^{m}(1-s)^{n-m}
$$

Given the individual state $\epsilon_{i}=\left(n_{i}, m_{i}, \sigma_{i}\right)$, player $i$ 's choice is $x_{i}=1$ with probability $y\left(n_{i}, m_{i}, \sigma_{i}\right)$, and zero otherwise. This implies an explicit formula for the probability that $x_{i}=1$, given the fraction $S_{i-1}$ of predecessors who played stock, the index $i$, the state $Z$, and the policy $y()$ :

$$
\begin{aligned}
& T_{i}\left(S_{i-1}, Z, y()\right) \equiv \operatorname{prob}\left(x_{i}=1 \mid i, S_{i-1}, Z, y()\right)= \\
& \sum_{n=0}^{N_{i}^{\text {max }}} N_{i}(n) \sum_{m=0}^{n} M_{i}\left(m \mid n, S_{i-1}\right) \int_{\sigma \in \mathcal{R}} y(n, m, \sigma) d F(\sigma \mid Z)
\end{aligned}
$$

Notice that for large $i, N_{i}^{\max }=N^{\max }, Q_{i}(n)=Q(n)$, and $M_{i}(m \mid n, s) \rightarrow$ $M(m \mid n, s)$, so the sequence of functions $T_{i}$ approaches a limit $T$ :

$$
T(s, z, y()) \equiv \lim _{i \rightarrow \infty} T_{i}(s, z, y())
$$

For each $n$ and $m$, the limiting distribution $M(m \mid n, S)$ is a smooth function of $S$, and the limiting function $T$ is just a weighted sum over the functions $M$. Therefore $T$ is a $C^{\infty}$ function of $S$.

This function $T$ helps us construct the probability distribution over aggregate outcomes $(S, Z)$. Consider the conditional probability $p_{i}(s \mid z, y())$ that $S_{i}$ takes some particular value $s$ in $\mathcal{J}_{i}$ :

$$
p_{i}(s, \mid z, y()) \equiv \operatorname{prob}\left(S_{i}=s \mid i, Z=z, y()\right)
$$

The number who play stock up to and including agent $i, X_{i}$, equals either $X_{i-1}$ or $X_{i-1}+1$, depending on whether or not $i$ plays stock. This means we can calculate $p_{i}(s \mid Z, y())$ recursively from $p_{i-1}(s \mid Z, y())$. For any $s \in \mathcal{J}_{i}$, we have: 


$$
\begin{aligned}
& p_{i}(s \mid Z, y())=p_{i-1}\left(\frac{i s}{i-1} \mid Z, y()\right) {\left[1-T_{i}\left(\frac{i s}{i-1}, Z, y()\right)\right] } \\
&+p_{i-1}\left(\frac{i s-1}{i-1} \mid Z, y()\right) T_{i}\left(\frac{i s-1}{i-1}, Z, y()\right)
\end{aligned}
$$

Given these probabilities, we can next define the corresponding cumulative distribution over $S$ and $Z$ :

$$
P_{i}(s, z \mid y()) \equiv \int_{-\infty}^{z} \sum_{S \leq s} p_{i}(S \mid Z, y()) d \zeta(Z)
$$

We will denote by $P$ the limit, as $i \rightarrow \infty$, of this $c . d . f$. :

$$
P(S, Z \mid y()) \equiv \lim _{i \rightarrow \infty} P_{i}(S, Z \mid y())
$$

What does the limiting distribution $P$ look like? The answer is made easier by the fact that $S_{i}$ is a stochastic recursive algorithm, in the sense of Evans and Honkapohja (2001). They show that under certain regularity conditions, a stochastic recursive algorithm converges with probability one to some stable fixed point of an associated ODE, a property they call "E-stability". In our case, the E-stability criterion simply means that the limit points of $S_{i}$ are points where $T$ crosses the $45^{\circ}$ line from above. ${ }^{3}$

Since $F$ has unbounded support, $T$ is above the $45^{\circ}$ line when $S=0$, and below it when $S=1$. In the generic case where $T$ is never tangent to the $45^{\circ}$ line, we can define $\Sigma(Z) \equiv\left\{S_{1}^{s}(Z), S_{1}^{u}(Z), S_{2}^{s}(Z), \ldots, S_{K(Z)}^{s}(Z)\right\}$ to be the $2 K(Z)-1$ points in $(0,1)$ at which $T$ crosses the $45^{\circ}$ line, conditional on a given $Z$. Define also the set of "stable" crossings, that is, crossings from above: $\Sigma^{s}(Z) \equiv\left\{S_{1}^{s}(Z), S_{2}^{s}(Z), \ldots, S_{K(Z)}^{s}(Z)\right\}$. Now although our model does not satisfy all the regularity conditions of Evans and Honkapohja, we can show the following result, using arguments from Fudenberg and Levine (1998).

\footnotetext{
${ }^{3}$ The function $h(\theta)$ defined on page 35 of Evans and Honkapohja (2001) corresponds to $T(S, .,)-$.$S in our notation.$
} 
Proposition 1. Suppose the policy function is $y^{*}$ and the aggregate state is $Z$, and that the function $T\left(S, Z, y^{*}\right)$ is never tangent to the $45^{\circ}$ line. Then with probability one, the limit of $S_{i}$ exists, and satisfies

$$
\lim _{i \rightarrow \infty} S_{i} \equiv S \in \Sigma^{s}(Z)
$$

Proof. See appendix.

Thus, conditional on the aggregate state $Z, S_{i}$ converges to one of a set of isolated points: the points $\Sigma^{s}(Z)$ where the $T$ function for that $Z$ crosses the $45^{\circ}$ line from above. For each $Z$, the c.d.f. $P(S, Z)$ is a step function, which increases only at the points $\Sigma^{s}(Z)$.

Given $P$, we can now calculate the distribution $\Pi$ that a player should attribute to $S$ and $Z$ conditional on her observations $(n, m, \sigma)$. Note that since agents know nothing about their positions in the sequence (except that $i>n_{i}$ ), the probability of being a member of any finite initial sequence $\mathcal{I}_{j}=\{1,2, \ldots j\}$ is negligible compared to the probability of being in the set $\{j+1, j+2, \ldots\}$. Thus players should ignore the probabilities $P_{i}$ and $M_{i}$ associated with being one of the first to choose, and consider only their limits $P$ and $M$. Using Bayes' law, if agents play $x=1$ with probability $y(n, m, \sigma)$, then rational expectations requires the following probability assessment:

$$
\Pi(z, s \mid n, m, \sigma)=\frac{\int_{(0,-\infty)}^{(s, z)} M(m \mid n, S) f(\sigma \mid Z) d P(S, Z \mid y())}{\int_{(0,-\infty)}^{(1, \infty)} M(m \mid n, S) f(\sigma \mid Z) d P(S, Z \mid y())}
$$

This probability information is all that an individual needs to choose optimally; and the individual's policy function, as we have just seen, suffices to construct this conditional distribution. Thus we can now define equilibrium.

Definition. A herding equilibrium is a policy function $y(n, m, \sigma)$ and a conditional probability distribution $\Pi(Z, S \mid n, m, \sigma)$ which satisfy these two conditions:

1. Given $\Pi$, policy $y$ solves the individual's problem (11).

2. Given $y$, distribution $\Pi$ satisfies the rational expectations condition (23). ${ }^{4}$

\footnotetext{
${ }^{4}$ Here the formula for $\Pi$ in equation (23) also depends on function $P$, as defined in (21), function $P_{i}$, as defined in (20), function $p_{i}$, as defined in (18), function $M$, as defined in (15), function $M_{i}$, as defined in (14), function $T$, as defined in (17), function $T_{i}$, as defined in (16), function $N$, as defined in (8), and function $N_{i}$, as defined in (7).
} 


\subsection{Computation}

As long as the aggregate state $Z$ takes only a finite number of values, the definition above offers a straightforward algorithm for calculating equilibrium.

0. Choose parameters $N^{m a x}, q, w^{s c}$, and $w^{\text {het }}$, and distributions $F$ and $\zeta$.

1. Guess a joint distribution $P(S, Z)$ over a fine grid of values $S \in[0,1]$ and $Z \in \mathcal{Z}$, such that the unconditional c.d.f. of $Z$ is $\zeta(Z)$.

2. Construct the conditional expectation $\Pi(Z, S \mid n, m, \sigma)$ implied by $P$, using equation (23).

3. For each $n$ and $m$, solve the utility maximization problem (11) over a fine grid of points $\sigma$. Interpolate to find the threshold signal(s) $\theta(n, m)$ where $x$ changes from 0 to 1 (or vice versa).

4. Construct the sequence of probability distributions $P_{i}(s \mid i, Z, y())$ implied by the policy $\theta(n, m)$, using equations (16)-(20). Continue until the sequence $P_{i}$ converges to a limiting distribution $P$.

5. Return to step (2). Iterate over steps (2)-(4) until fixed points for the distribution and policy function are found. ${ }^{5}$

Multiple equilibria are possible in this model, especially if strategic complementarities are strong, so this algorithm could converge to more than one fixed point depending on the distribution $P$ from which we start. Here we focus on the equilibrium to which we converge from a uniform prior $P$. Although we are not explicitly analyzing learning, it is reasonable to suppose that this is the equilibrium which would be learned through repeated play, starting from a situation without information. ${ }^{6}$

\footnotetext{
${ }^{5}$ To ensure convergence, it is usually necessary to allow only partial adjustment when we update the thresholds $\theta$ and distributions $P$ in steps (3) and (4).

${ }^{6}$ This assumes that, like our algorithm, the learning process relies on relatively slow partial adjustment to guarantee convergence.
} 


\section{Information and strategic complementarities in herding equilibrium}

We now compute some examples of herding equilibria, focusing especially on the role of strategic complementarities. In the reported results, the functions $\theta(n, m)$ and $P(S, Z)$ both exhibit errors of less than $10^{-6}$ at all points.

\subsection{Discrete distribution of $Z$}

For ease of computation, we start by allowing just two possible aggregate states, $Z=0$ and $Z=1$, with probability 0.5 each. We assume in all our simulations that the signal $\sigma$ is normally distributed with conditional mean $Z-0.5$ and conditional standard deviation $\eta$. In this subsection we set $\eta=1$. We set $N^{\max }=20$ and $q=0.9$, so that on average, players observe 18 previous actions. We study what happens as strategic complementarities increase, setting $w^{h e t}=0.1, w^{s c}=0.3,0.5,0.7$, or 0.9 , and as always, $w^{\text {info }}=1-w^{\text {het }}-w^{s c}$.

Proposition 1 showed that we can learn a lot from the shape of the function $T$ defined in (16) and (17). If $y^{*}$ is the equilibrium policy, and if the equation

$$
S=T\left(S, Z, y^{*}\right)
$$

crosses the $45^{\circ}$ line from above at some point $S^{*}$, then there is positive probability of a history in which $\lim _{i \rightarrow \infty} S_{i}=S^{*}$. Figure 1 shows how the functions $T\left(S, 0, y^{*}\right)$ and $T\left(S, 1, y^{*}\right)$ change as we vary $w^{s c}$. The mapping for good times $(Z=1)$ is shown as a solid line, and that for bad times $(Z=0)$ as a dashed line; we also show the $45^{\circ}$ line.

We see two very different regimes of behavior. If the herding incentive comes mostly from information revelation (cases $w^{s c}=0.3$ and 0.5 ), then $T$ bulges out around the $45^{\circ}$ line, with a single stable crossing for each $Z$, at $S \approx 0$ for $Z=0$ and at $S \approx 1$ for $Z=1$. Function $T$ bends back towards the $45^{\circ}$ line at the opposite end, but does not cross again, so play should converge to a single outcome $S(Z)$ for each $Z$. On the other hand, for higher strategic complementarities, $w^{s c}=0.7$ or 0.9 , the $T$ function changes dramatically. It 
is now S-shaped, with two stable crossings, close to zero and one, for each $Z$. This means herding can go "wrong" with positive probability, converging to more than one outcome in each state $Z$.

We observe the process of convergence in Figures 2 and 3. Figure 2 plots the simulated cumulative distribution $\operatorname{prob}\left(S_{300} \leq s \mid Z, y^{*}\right)$ as a function of $s$, for each $Z$ and for each value of $w^{s c}$ considered. That is, we plot the distribution, across histories, of the fraction of individuals who have chosen $x=1$, up to and including the 300th individual. ${ }^{7}$ The c.d.f. of $S_{300}$ conditional on $Z=0$ lies to the left of (or above) the c.d.f. conditional on $Z=1$ : as expected, a low $S$ is more likely when $Z=0$.

If $w^{s c}=0.3$ or 0.5 , then as of $i=300$, roughly $60 \%$ of histories have converged to the "right" aggregate outcome. With probability 0.3 , the outcome is $Z=0$ and $S_{300} \approx 0$; and with probability 0.3 , it is $Z=1$ and $S_{300} \approx 1$. But in the remaining histories, $S_{300}$ is spread almost uniformly between 0 and 1 . Figure 3 shows a sample of 200 histories of length 500 for the case $w^{s c}=0.5$, conditional on $Z=1.8$ Here we see that $S_{i}$ sometimes converges almost immediately to one (there is a corresponding spike in the density function, plotted below). But other histories start near zero and tend slowly upwards, and are still widely spread out as of $i=500$.

In contrast, for $w^{s c} \geq 0.7$, Figure 2 shows that $S_{300}$ is almost always near 0 or 1 . The outcome $Z=0$ and $S_{300} \approx 0$ occurs around $35 \%$ of the time, while the outcome $Z=0$ and $S_{300} \approx 1$ occurs $15 \%$ of the time; for $Z=1$ the probabilities are reversed. Thus, strong strategic complementarities imply average play converges quickly, but may converge to the wrong action. This quick convergence is also seen in Figure 3, where in a sample of 200 histories, all but one are clearly trending up or down by step $i=10$.

In all these simulations, 18 predecessors are observed on average, and the limiting $S$ is close to 0 or 1 , so most players can easily infer the true $S$. When

\footnotetext{
${ }^{7}$ In the graph, the maximum probability shown is $50 \%$, because we have scaled by the unconditional probability of $Z$.

${ }^{8}$ Less than 200 lines are visible, since some histories sometimes lie exactly on top of others.
} 
$w^{s c}$ is small, $S$ is uniquely linked to $Z$, so $Z$ is inferred too. However, this also means that for low $w^{s c}$, a few agents are sometimes totally deceived, especially near the beginning of the sequence. If the first few players take the "wrong" action, their successors are likely to place high probability on the wrong $Z$ and to repeat their error. This is why $T$ curves back towards the $45^{\circ}$ line at both ends, and wrong outcomes $S_{i}$ are persistent, as Figures 1-3 show. On the other hand, with stronger strategic complementarities, players know that the $S$ they observe is often inconsistent with the true $Z$. Given high $w^{s c}$, the incentive to mimic others outweighs the incentive to choose in accordance with the true state, so the "wrong" outcome is self-fulfilling even in the infinite limit.

\subsection{Continuous distribution of $Z$}

While our conclusions thus far make sense, we should check whether they change if we alter the distribution of $Z$. In particular, we should consider a continuous support for $Z$, since this is the usual assumption in the global games literature, and may imply a substantially different inference problem. Thus we next assume that $Z$ is distributed $N\left(\frac{1}{2}, \frac{1}{6}\right)$ (so as before, $Z$ is usually between 0 and 1). We approximate this case by making the support $\mathcal{Z}$ a grid of 151 points from -1 to 2 , with steps of 0.02 . We assume $\sigma$ is conditionally normal with mean $Z-0.5$ and standard deviation $\eta=0.1$, and we set $N^{\max }=10$ and $q=0.9$. We compare the cases $w^{s c}=0.3$ and $w^{s c}=0.6$.

Figure 4 shows the results, which generalize our previous conclusions in an intuitive way. The graphs now show 151 different $T$ functions simultaneously, one for each grid point of $\mathcal{Z}$. In the center of each graph we see the S-shape of $T$ that occurs near $Z=0.5$, with multiple crossings for both $w^{s c}=0.3$ and $w^{s c}=0.6$. Since the stepsize for $Z$ is 0.02 , we can conclude from the graph that with low strategic complementarities $\left(w^{s c}=0.3\right)$, multiple outcomes occur from roughly $Z=0.34$ to $Z=0.66$ (around one standard deviation of $Z$ ). For $w^{s c}=0.6$, there are multiple outcomes from $Z=-0.6$ to $Z=1.6$.

In both graphs, as we move away from $Z=0.5$, function $T$ arcs outwards from the $45^{\circ}$ line, almost but not quite crossing twice. As before, this implies 
persistent initial "errors", but eventual convergence to a unique outcome consistent with $Z$. For more extreme values of $Z, T$ becomes almost flat, crossing the $45^{\circ}$ line just once and implying quick convergence to the "right" outcome.

Thus when $Z$ has continuous support, the set of aggregate states divides into three regions: for low $Z$, there is just one outcome, with $S \approx 0$; around $Z=0.5$ there are multiple outcomes; and for high $Z$ the outcome is uniquely $S \approx 1$. Stronger strategic complementarities make the interval of multiplicity wider, but it is nonempty even with relatively low $w^{s c}$. This "tripartite classification of fundamentals" (Morris and Shin 1998) is familiar from the context of currency crises under full information. Also, Figure 4 helps us reinterpret the case of binary $Z$ studied in Section 3.1: both $Z=0$ and $Z=1$ lie inside the zone of multiple outcomes if $w^{s c}=0.6$, but outside it if $w^{s c}=0.3$.

\section{Alternative information structures}

Our purpose here is to compare the implications of herding to those of fullinformation games and global games. Thus, suppose next that $Z$ and $S$ are perfectly observed. Equilibrium then requires optimal choice, given $Z$ and $S$ :

$$
y(\sigma, S, Z) \in \underset{y \in[0,1]}{\operatorname{argmax}} y u(1, Z, S, \sigma)+(1-y) u(0, Z, S, \sigma)
$$

and it also requires that $S$ results from aggregating choices $y$ :

$$
S=\int_{\sigma \in \mathcal{R}} y(\sigma, S, Z) d F(\sigma \mid Z)
$$

Equations (25) and (26) define a best response function $s=T^{f i}(S, Z)$ which states the fraction $s$ of agents who would play "stock" conditional on the information that the fraction of agents playing stock were $S$. Thus the points where $T^{f i}$ crosses the $45^{\circ}$ line for given $Z$ are the outcomes $S(Z)$ that occur in full-information equilibrium. Equivalently, we can define a full-information equilibrium as a pair $\left(y^{f i}(\sigma, Z), S^{f i}(Z)\right)$ that solve (25) and (26). 
Notice that under full information, the idiosyncratic shock $\sigma$ adds heterogeneity to payoffs only, not to information. This means that with full information, stronger strategic complementarities favor multiplicity of equilibrium, while greater heterogeneity of $\sigma$ favors uniqueness. Specifically: ${ }^{9}$

Proposition 2. a. Holding fixed the distribution $F$ and the parameter $w^{\text {het }}$, there is a unique full information equilibrium if $w^{s c}$ is sufficiently close to zero, and multiplicity if $w^{s c}$ is sufficiently close to one.

b. Suppose $F$ is normal, with conditional standard deviation $\eta$ and conditional mean such that a player with the mean $\sigma$ would change his choice $x$ if all others did so too. Fixing other parameters, there is a unique full information equilibrium if $\eta$ is sufficiently large, and multiplicity if $\eta$ is sufficiently small.

Proof. See appendix.

In the appendix we calculate bounds on these equilibria, using the indifference condition (42) and the fact that $1 \%$ of the normal distribution lies more than 2.33 standard deviations above its mean. We find that there is an equilibrium with $S>0.99$ if and only if $Z>\frac{1}{2}+z^{*}$, and an equilibrium with $S<0.01$ if and only if $Z<\frac{1}{2}-z^{*}$, where $z^{*} \approx\left(0.49 w^{s c}-2.33 w^{\text {het }} \eta\right) /\left(w^{\text {info }}+w^{\text {het }}\right)$. As in the proposition, greater $w^{\text {het }}$ or $\eta$ shrinks the region of multiplicity, while greater $w^{s c}$ expands it. For example, if $\eta=0.1, w^{\text {het }}=0.1$, and $w^{s c}=0.3$, then $z^{*} \approx 0.18$, matching almost perfectly the range of multiple herding outcomes we found for these parameters (see Section 3.2 and Figure 4). But if $w^{s c}=0.6$, then $z^{*} \approx 0.68$, so for these parameters the interval $Z \in[-0.6,1.6]$ of multiple herding outcomes is substantially larger than the interval $Z \in[-0.18,1.18]$ of full-information multiple equilibria. In herding equilibrium, one follows the crowd not only because of strategic complementarities, but also because others' actions reveal information about the aggregate state. This helps widen the range of multiplicity in herding equilibrium.

Since there may exist multiple full information equilibria, we can also define sunspot equilibria in which we "flip a coin" to decide which equilibrium is played. That is, suppose there are $K$ full information equilibria

\footnotetext{
${ }^{9}$ These results are analogous to those in Ciccone and Costain (2001).
} 
$\left(y_{k}^{f i}(\sigma, Z), S_{k}^{f i}(Z)\right)$ indexed by $k \in\{1,2, \ldots K\}$. Choose any random variable $\xi$ with support $\Xi$, and a function $k(\xi)$ mapping $\Xi$ into $\{1,2, \ldots K\}$. Then we define a sunspot equilibrium as $y^{s s}(\sigma, \xi) \equiv y_{k}^{f i}(\sigma)$ and $S^{s s}(Z, \xi) \equiv S_{k}^{f i}(Z)$ whenever $k(\xi)=k$. The $c$.d.f. over outcomes $(S, Z)$ in this sunspot equilibrium will be called $P^{s s}(S, Z)$.

The global games framework generalizes our full information game by allowing informational heterogeneity across agents. Here, if $Z$ and $S$ are not observed, but must instead be inferred only from the signal $\sigma$, then our model is a global game. A global games equilibrium is a pair $\left(y^{g g}(\sigma), S^{g g}(Z)\right)$ such that choice is optimal:

$y^{g g}(\sigma) \in \underset{y \in[0,1]}{\operatorname{argmax}} \int_{Z \in \mathcal{Z}}\left[y u\left(1, Z, S^{g g}(Z), \sigma\right)+(1-y) u\left(0, Z, S^{g g}(Z), \sigma\right)\right] \frac{f(\sigma \mid Z) d \zeta(Z)}{\int_{Z^{\prime} \in \mathcal{Z}} f\left(\sigma \mid Z^{\prime}\right) d \zeta\left(Z^{\prime}\right)}$

and $S^{g g}$ results from aggregating choices $y^{g g}$ :

$$
S^{g g}(Z)=\int_{\sigma \in \mathcal{R}} y^{g g}(\sigma) d F(\sigma \mid Z)
$$

It is clear from (27) and (28) that our herding equilibrium, in turn, is a generalization of a global games equilibrium, in which players observe a sample other players' actions in addition to the signal.

The global games literature claims that even infinitesimal heterogeneity of information often leads to uniqueness. In our framework, we can show:

Proposition 3. Let utility $u$ be given by (5). Let the distribution of $\sigma$ be normal, with conditional mean $Z-0.5$ and conditional variance $\eta$. Then:

a. If $Z$ takes values 0 or 1 with probability 0.5 each, then as $\eta \rightarrow 0$, there are multiple global games equilibria if and only if $w^{s c} \geq w^{\text {het }}+w^{\text {info }}$.

b. If $Z$ is normally distributed, then if $\eta$ is sufficiently small but strictly positive, there is a unique global games equilibrium in which $y^{g g}(\sigma)=1$ if and only if $\sigma$ is positive. 


\section{Proof. See appendix.}

This proposition reminds us that global games do not always imply uniqueness. With a binary state space $\mathcal{Z}$, our model's behavior is qualitatively the same regardless of whether we treat it as a herding game, as a full-information game, or as a global game. But when $Z$ is normally distributed (so that $\mathcal{Z}$ is a continuum) both the herding game and the full-information game exhibit an interval of multiple outcomes, while the full-information limit of the global game has a unique equilibrium and hence a unique outcome $S^{g g}(Z)$. In other words, it is precisely when the famous global games uniqueness argument is applicable that the herding model's predictions are different. Just as the fullinformation conclusions appear fragile to imperfect information, the global game conclusions appear fragile to observations of other players' choices.

\section{The full information limits of the herding model}

While it now appears that the herding model behaves more like the fullinformation game than the global game, we must remember that the global games uniqueness result relies on being sufficiently close to full information. Thus to clarify the relation between the three games, we should also investigate what occurs in our herding model as we approach full information.

In a global game, one approaches full information by decreasing the standard deviation $\eta$ of the signal. But in our model, there is also a second route to full information, which arises neither in the global games setup nor in a standard herding model: we also approach full information if we increase $N^{\max }$ without bound, revealing ever more accurate information to ever more agents. Here we study the implications of both full information limits.

\subsection{The perfect signals limit}

Figure 5 shows what happens as the signal's precision (the inverse of its standard deviation $\eta$ ) varies from 0.03 to 10 . (We set $w^{s c}=0.7$, implying strong 
strategic complementarities, with other parameters as in Section 3.1.) Low precision (high variance) means there is little information in the signal. However, since $\sigma$ enters directly in the utility function, high variance also means very strong preferences over $x$ : for many agents, $\sigma$ is so large or so small that the utility component $u^{\text {het }}(x, \sigma)$ dominates, and they hardly care what others do. Hence for precision $\eta^{-1}=0.03$, the function $T$ is almost flat, and the outcome depends uniquely on $Z$.

Gradually, as we increase precision, individuals respond more to their observations of others. With precision $\eta^{-1}=0.3$, the outcome is still unique, but there are persistent errors. With higher precision, multiple outcomes occur with positive probability. Even though each individual's signal is quite accurate, people condition their choices on others' play, both because they know that others' signals are improving too, and also because of strategic complementarities. Thus, we have multiple outcomes as we approach full information, and in this sense the standard global games limiting result fails in our model.

On the other hand, Figure 6 shows that "wrong" outcomes become less and less likely as we approach full information. Though erroneous behavior is persistent, high signal accuracy implies that the players who choose first usually make the right decision, and the probability of herding to the wrong outcome approaches zero. Therefore, we might reasonably argue that the existence of multiplicity in this limit is not, by itself, very relevant. In this sense, the global games limiting result is partially confirmed by our model.

\subsection{The large numbers limit}

Figures 7 and 8 analyze the effects of changing $N^{\max }$ from 2 to 80 . We set the standard deviation of $\sigma$ to $\eta=1$, leaving other parameters as before. Since $q=0.9$, the average number of observations goes from 1.8 to 72 , which suffices to show us the effects of an extremely accurate estimate of $S$.

For $N^{\max }=2$, there is always a unique outcome close to 0.5 , because with so few observations there is little incentive to herd. Individuals mainly follow their own signals, so by the law of large numbers the outcome is unique, 
and we observe a narrow distribution of $S_{300}$ in Figure 8. Increasing $N^{\max }$ to 5, the outcome is still unique, but "errors" are persistent - now there are sufficient observations to justify following others. Figure 8 shows that for this case, roughly $70 \%$ of histories converge quickly to the right outcome, but the remainder are still widely dispersed as of $i=300$.

At all higher levels of $N$, there are multiple outcomes for each $Z$. Almost all histories have almost converged to $S_{i} \approx 0$ or $S_{i} \approx 1$ by $i=300$, and the convergence gets faster with larger $N^{\max }$. Strong strategic complementarities mean people closely follow the crowd, so there is multiplicity in spite of the excellent information. Moreover, Figure 8 shows that the probability of the wrong outcome is almost exactly the same, independent of $N^{\max }$, for all $N^{\max } \geq 10$. This suggests that the probability of various outcomes is mostly determined by the distribution of events that can occur in the first few steps. ${ }^{10}$

These numerical results demonstrate that if players have a large, or even moderate (like 10) sample of observations, multiple outcomes occur with nontrivial probability. In this sense, our herding model contradicts the global games uniqueness result and instead behaves more like a sunspot equilibrium of the full information game. To further investigate the relation with sunspots, let $y^{*}$ be the equilibrium strategy and consider how the function $T\left(S, Z, y^{*}\right)$ changes as $N^{\max }$ grows large. Let $T^{\infty}(S, Z)$ be its limit as $N^{\max } \rightarrow \infty$. Similarly, let $P^{\infty}(S, Z)$ be the limit of the equilibrium c.d.f. $P\left(S, Z \mid y^{*}\right)$ as $N^{\max } \rightarrow \infty$. With this notation, we can show that our herding equilibrium converges in distribution to a full-information sunspot equilibrium.

\footnotetext{
${ }^{10}$ We draw two interesting conclusions. First, making small changes in the distribution of observations - for example, imposing a different function $N(n)$ with the same mean, or assuming sampling with replacement, instead of without replacement - should change the probability distribution over aggregate outcomes. This appears to be another way our model differs from global games, where a "noise-independent selection" result sometimes applies (Carlsson and van Damme 1993; Frankel, Morris, and Pauzner, 2003).

Second, we conclude that allowing players to observe each previous choice with constant probability - instead of bounding the number of observations - would not qualitatively change our results. Most agents would have many observations, but their policy functions would be similar to those calculated here.
} 
Proposition 4. Fix all parameters except $N^{\max }$, and consider the limit of the herding equilibrium as $N^{\max } \rightarrow \infty$. Assume that $F$ contains no point masses, but that $\mathcal{Z}$ is discrete. Then the limiting c.d.f. $P^{\infty}(S, Z)$ of the herding model is equal to the c.d.f. $P^{s s}(S, Z)$ generated by some sunspot equilibrium of the full-information model.

Proof. See appendix.

A corollary of this proposition is that if the full information game has multiple equilibria, then for some $N^{*}, N^{\max }>N^{*}$ implies the existence of a herding equilibrium which exhibits multiple outcomes.

The intuition behind this proposition is just that large $N^{\max }$ reveals the true state almost perfectly. Thus, at the equilibrium outcomes of the herding model, most players' thresholds are close to those of the full-information policy. This means points where the limiting $T^{\infty}(S, Z)$ of the herding model crosses the $45^{\circ}$ line must also be points where the full-information $T^{f i}(S, Z)$ is on the $45^{\circ}$ line. Hence the limiting distribution $P^{\infty}(S, Z)$, like a sunspot equilibrium, randomizes over outcomes which are equilibria of the full information game. But unlike sunspot equilibria in general, the sunspot equilibrium selected by $P^{\infty}(S, Z)$ in our simulations is well-behaved, exhibiting a positive correlation between fundamentals $Z$ and outcomes $S$.

\section{Conclusions}

The global games literature has shown that multiple equilibria often vanish if information is slightly heterogeneous; this suggests that we should expect uniqueness in practice, since full information is clearly an unrealistic special case. But global games have themselves usually been constructed in an unrealistic special case: that of exactly simultaneous decision. Given a situation like a bank run, it seems absurd to suppose that most people choose before observing any other choices. Instead, most people will observe a random subset of predecessors: exactly the structure this paper models. In such an environment, the "tripartite classification" of aggregate states, including an interval of multiple outcomes, is a robust phenomenon. The region of multiplicity is 
larger if strategic complementarities are stronger, and tends to be at least as large as the region of multiple full-information equilibria.

We have seen that approaching full information by increasing signal quality drives the probability of the wrong herding outcome to zero, partially confirming the global games uniqueness result. But it seems unlikely, in the economic contexts we have mentioned, that everyone has almost perfect private information about the true state. ${ }^{11}$ Instead, it makes sense to suppose that choice is not exactly simultaneous, and therefore that some previous choices are observed. When we approach full information by increasing the average number of observations, a high probability of reaching the wrong aggregate outcome remains. In fact, numerically, the probability distribution over aggregate outcomes hardly varies with $N^{\max }$ for $N^{\max } \geq 10$. One of the many ways our herding model resembles the full-information game is that with a discrete aggregate state space, this probability distribution equals that associated with one of the well-behaved full-information sunspot equilibria.

Several important but possibly difficult extensions come to mind. Future research should relax the assumption of a binary choice set, and allow for prices (endogenous public signals). It would also be interesting to study dynamics, perhaps just by repeating the game analyzed here. Alternatively, we could reinterpret the timing of the sequence, assuming that choices take place over a long horizon. It would then be more natural to link observations and payoffs to nearby players in the sequence; and we would expect to observe dynamics that usually hovered near some stable outcome, with occasional random transitions from one stable outcome to another.

Thus we have still not resolved the debate over the uniqueness or multiplicity of the mapping between fundamentals and outcomes. However, this paper strongly suggests that global games have nothing to contribute to the debate. The complicated iterated dominance reasoning about average play that underlies the global games uniqueness result is easily short-circuited by

\footnotetext{
${ }^{11}$ Hellwig (2002) shows it is essential for the global games uniqueness result that the signal be private information; hence $\sigma$ cannot be interpreted as a price.
} 
a few observations of others. Perhaps the global games result still has a role as a technical trick when the possibility of multiplicity is not the question at hand. But we should not suppose that the global games uniqueness result is an empirical prediction. Nor should we attempt to draw policy conclusions about how to prevent multiplicity by studying global games.

\section{References}

Banerjee, Abhijit (1992), "A Simple Model of Herd Behavior." Q.J.E. 107 (3), pp. 797-818.

Bikhchandani, Sushil; David Hirshliefer, and Ivo Welch (1992), "A Theory of Fads, Fashion, Custom, and Cultural Change as Informational Cascades." J.P.E. 100 (5), pp. 992-1026.

Bikhchandani, Sushil; David Hirshliefer, and Ivo Welch (1996), "Informational Cascades and Rational Herding: An Annotated Bibliography." Available at: http://welch.som.yale.edu/cascades/

Bikhchandani, Sushil; David Hirshliefer, and Ivo Welch (1998), "Learning from the Behavior of Others: Conformity, Fads, and Informational Cascades." J.E.P. 12 (3) pp. 151-70.

Cabrales, Antonio; Rosemarie Nagel, and Roc Armenter (2002), "Equilibrium Selection through Incomplete Information in Coordination Games: an Experimental Study." Univ. Pompeu Fabra Economics Working paper \#601.

Carlsson, Hans and Eric van Damme (1993), "Global Games and Equilibrium Selection." Econometrica 61 pp. 989-1018.

Chamley, Christophe, Rational Herds: Economic Models of Social Learning, forthcoming 2003, Cambridge University Press.

Chamley, Christophe (2002), "Social Learning through the Observation of Discrete Choices is Very Slow." Manuscript, Boston Univ.

Chari, V.V., and Patrick Kehoe (2002), "On the Robustness of Herds." Federal Reserve Bank of Minneapolis Working Paper 622.

Ciccone, Antonio, and James Costain (2001), "On Payoff Heterogeneity in Games with Strategic Complementarities." Univ. Pompeu Fabra Economics Working Paper \#546.

Cooper, Russell, and Andrew John (1988), "Coordinating Coordination Failures in Keynesian Models." Q.J.E. 103, pp. 441-63. 
Evans, George W., and Seppo Honkapohja (2001), Learning and Expectations in Macroeconomics, Princeton University Press.

Frankel, David M., Stephen Morris, and Ady Pauzner (2003), "Equilibrium Selection in Global Games with Strategic Complementarities." J.E.T. 108, pp. 1-44.

Fudenberg, Drew, and David K. Levine (1998), The Theory of Learning in Games, MIT Press.

Heinemann, Frank; Rosemarie Nagel; and Peter Ockenfels (2002), "Speculative Attacks and Financial Architecture: Experimental Analysis of Coordination Games with Public and Private Information." Manuscript, LudwigMaximilians-Universitat Munchen.

Hellwig, Christian (2002), "Public Information, Private Information, and the Multiplicity of Equilibria in Coordination Games." J.E.T. (107), pp. 191222.

Herrendorf, Berthold; Akos Valentinyi, and Robert Waldmann (2000), "Ruling out Multiplicity and Indeterminacy: the Role of Heterogeneity." R.E.Stud. 67, pp. 295-307.

Morris, Stephen, (2000), "Contagion." R.E.Stud. 67, pp. 57-78.

Morris, Stephen, and Atsushi Kajii (1997), "The Robustness of Equilibrium to Incomplete Information." Econometrica 65, pp. 1283-1309.

Morris, Stephen, and Hyun Song Shin (1998), "Unique Equilibrium in a Model of Self-Fulfilling Currency Attacks." A.E.R. 88 (3), pp. 587-97.

Morris, Stephen, and Hyun Song Shin (2003), "Heterogeneity and Uniqueness in Interaction Games." Cowles Foundation Discussion Paper \#1402, Yale Univ.

Perktold, Josef (1996) "Recurring Informational Cascades." Manuscript, Univ. of Chicago.

Schmitt-Grohe, Stephanie, and Martin Uribe (2001), "On the Perils of Taylor Rules." J.E.T. 96 pp. 40-69.

Schmutzler, Armin (1998), "Changing Places: The Role of Heterogeneity and Externalities in Cumulative Processes." International Journal of Industrial Organization, 16, pp. 445-461.

Smith, Lones, and Peter Sorensen (2002), "Pathological Outcomes of Observational Learning." Econometrica, (68), pp. 371-98.

Vives, Xavier (1993), "How Fast Do Rational Agents Learn?" R.E.Stud., (60), pp. 329-347.

Woodford, Michael (1990), "Learning to Believe in Sunspots." Econometrica, 58 (2), pp. 277-307. 


\section{Appendix: Proofs}

\section{Proof of Proposition 1.}

This proof is an extension of Fudenberg and Levine (1998), pp. 131-3.

We assume $T$ is never tangent to the $45^{\circ}$ line. Define the set of unstable crossings of $T$, augmented by corners: $\Sigma^{u}(Z) \equiv\left\{0, S_{1}^{u}(Z), \ldots, S_{K(Z)-1}^{u}(Z), 1\right\}$. We refer to the $K(Z)$ intervals between elements of $\Sigma^{u}(Z)$ as the basins of attraction for the $K(Z)$ stable steady states in $\Sigma^{s}(Z)$. We define the random variable $S_{i}^{*}$ to represent the stable steady state in the basin of attraction where $S_{i}$ currently lies. In other words, $S_{i}^{*} \equiv S_{k}^{s}$ if $S_{i}$ is in the $k$ th basin of attraction.

Let $D(x, y) \equiv(x-y)^{2}$ be the squared distance between two points, and let $L$ be the squared distance from the steady state in the current basin of attraction:

$$
L\left(S_{i}\right) \equiv D\left(S_{i}, S_{i}^{*}\right)=\left(S_{i}-S_{i}^{*}\right)^{2}
$$

Note that $L$ is bounded between 0 and 1 .

In general, the distances from a given unstable steady state $S_{i}^{u}(Z) \in \Sigma^{u}(Z)$ to the stable steady states above and below it may be different. This means that $L$ is in general discontinuous at the unstable steady states. To avoid complications arising from this discontinuity, define a step function $A(S)$ such that $A(0)=0$ and such that

$$
\hat{L}(S) \equiv L(S)+A(S)
$$

is a continuous function. Note that $A$ is uniquely defined, and is constant in each of the basins of attraction. That is, $\hat{L}$ is quadratic over each basin of attraction, with minima at each stable steady state $S^{s} \in \Sigma^{s}(Z)$, and with intercept terms adjusted so that $\hat{L}$ is continuous (but with a nondifferentiable peak) at each unstable steady state $S^{u} \in \Sigma^{u}(Z)$.

Let $G$ and $\hat{G}$ be the expected changes in $L$ and $\hat{L}$ :

$$
G\left(S_{i}\right) \equiv E\left(L\left(S_{i+1}\right) \mid S_{i}\right)-L\left(S_{i}\right) \quad \text { and } \quad \hat{G}\left(S_{i}\right) \equiv E\left(\hat{L}\left(S_{i+1}\right) \mid S_{i}\right)-\hat{L}\left(S_{i}\right)
$$

Now note that the expected squared distance of $S_{i+1}$ from the current stable steady state $S_{i}^{*}$ can always be calculated explicitly:

$$
\begin{gathered}
E\left(D\left(S_{i+1}, S_{i}^{*}\right) \mid S_{i}\right)-D\left(S_{i}, S_{i}^{*}\right)=T_{i}\left(S_{i}, Z\right)\left[\frac{i S_{i}+1-(i+1) S_{i}^{*}}{i+1}\right]^{2}+\left(1-T_{i}\left(S_{i}, Z\right)\right)\left[\frac{i S_{i}-(i+1) S_{i}^{*}}{i+1}\right]^{2}-\left(S_{i}-S_{i}^{*}\right)^{2} \\
=\frac{2 i\left(S_{i}-S_{i}^{*}\right)\left(T_{i}\left(S_{i}, Z\right)-S_{i}\right)+T_{i}\left(S_{i}, Z\right)-S_{i}^{2}+2 S_{i}^{*}\left(S_{i}-T_{i}\left(S_{i}, Z\right)\right)}{(i+1)^{2}} \\
\leq \frac{2 i\left(S_{i}-S_{i}^{*}\right)\left(T_{i}\left(S_{i}, Z\right)-S_{i}\right)+3}{(i+1)^{2}}
\end{gathered}
$$


The fact that $T$ crosses the $45^{\circ}$ line from above at a stable steady state implies that the term $\left(S_{i}-S_{i}^{*}\right)\left(T_{i}\left(S_{i}, Z\right)-S_{i}\right)$ is nonpositive, and strictly negative away from steady states.

Now as long as $S_{i+1}^{*}=S_{i}^{*}$ with probability one, then both $G$ and $\hat{G}$ are equal to $E\left(D\left(S_{i+1}, S_{i}^{*}\right) \mid S_{i}\right)-D\left(S_{i}, S_{i}^{*}\right)$, as given by (32). Moreover, if the distance from $S_{i}$ to the nearest unstable steady state is greater than $1 /(1+i)$, then $S_{i+1}^{*}=S_{i}^{*}$ with probability one. On the other hand, if there is positive probability, conditional on $S_{i}$, that $S_{i+1}$ will jump into a new basin of attraction (so that $S_{i+1}^{*} \neq S_{i}^{*}$ ), then since $\hat{L}$ has a sharp peak at the unstable steady state, $\hat{G}$ will be strictly less than the expression in (32). Thus, for all $S_{i}$, we have

$$
\hat{G}\left(S_{i}\right) \leq E\left(D\left(S_{i+1}, S_{i}^{*}\right) \mid S_{i}\right)-D\left(S_{i}, S_{i}^{*}\right) \leq \frac{2 i\left(S_{i}-S_{i}^{*}\right)\left(T_{i}\left(S_{i}, Z\right)-S_{i}\right)+3}{(i+1)^{2}}
$$

Note that the positive part of $\hat{G}$ is summable with respect to $i$. That is,

$$
\sum_{i=1}^{\infty} \hat{G}_{i} \leq \sum_{i=1}^{\infty} \max \left\{\hat{G}_{i}, 0\right\} \leq \sum_{i=1}^{\infty} \frac{3}{(1+i)^{2}}<\infty
$$

Now define

$$
\tilde{H}_{i} \equiv \hat{L}\left(S_{i}\right)-\sum_{j=1}^{i-1} \max \left\{\hat{G}\left(S_{i}\right), 0\right\}
$$

Note that $\tilde{H}$ is a supermartingale by construction, and from (34) we see that it is bounded below. Hence, by the martingale convergence theorem, the $\operatorname{limit}_{\lim _{i \rightarrow \infty}} \tilde{H}_{i}$ exists and is bounded below. Likewise, $\hat{L}\left(S_{i}\right)-\tilde{H}_{i}=$ $\sum_{j=1}^{i-1} \max \left\{\hat{G}\left(S_{j}\right), 0\right\}$ is a submartingale which is bounded above, and therefore converges. Since both $\tilde{H}_{i}$ and $\hat{L}\left(S_{i}\right)-\tilde{H}_{i}$ converge, we can conclude that $\hat{L}\left(S_{i}\right)$ converges too.

Now note that if $\hat{L}\left(S_{i}\right)$ converges, then $L\left(S_{i}\right)$ and $S_{i}$ must converge too. We will now show that if $S_{i}$ converges, it must converge to a steady state; thereafter we extend the argument to show that $S_{i}$ cannot converge to an unstable steady state.

Suppose that $S_{i}$ can converge with positive probability to a point which is not a steady state; then there is positive probability that $\lim _{i \rightarrow \infty} L\left(S_{i}\right)>0$. Then we can choose $I$ large enough so that for $i>I, S_{i}$ remains in only one basin of attraction, on one side of $S_{i}^{*}$. Let the distance between the stable steady state $S_{i}^{*}$ and the relevant unstable steady state be $l$. Then more specifically, $I$ can be chosen large enough so that we can choose some small $\epsilon_{1}>1 /(1+I)$ such that $\epsilon_{1}<\left|S_{i}-S_{i}^{*}\right|<l-\epsilon_{1}$. Now since $T$ is smooth, and since $\left(S_{i}-S_{i}^{*}\right)\left(T_{i}\left(S_{i}\right)-S_{i}\right)$ is strictly negative away from steady states, for 
$i>I$ there exists $\epsilon_{2}>0$ such that $\left(S_{i}-S_{i}^{*}\right)\left(T_{i}\left(S_{i}\right)-S_{i}\right)<-\epsilon_{2}$. Therefore for $i>I,(32)$ becomes

$$
G\left(S_{i}\right)=\hat{G}\left(S_{i}\right)<\frac{-2 i \epsilon_{2}+3}{(i+1)^{2}}
$$

The negative part of this expression is not summable with respect to $i$; therefore if $L$ converges to a positive number less than $l$, we have

$$
\sum_{j=1}^{\infty} \hat{G}\left(S_{j}\right)=-\infty
$$

Now define the martingale

$$
\hat{H}_{i}=\hat{L}\left(S_{i}\right)-\sum_{j=1}^{i-1} \hat{G}\left(S_{j}\right)
$$

Notice that from our previous calculation, $\hat{H}_{i} \geq \tilde{H}_{i} \geq 1-\sum_{i=1}^{\infty} \frac{3}{(1+i)^{2}}>-\infty$. Since $\hat{H}$ is a martingale, and bounded below, it converges to a limit which is bounded below. But if there is a positive probability that $\hat{L}$ converges to a positive number less than $l$, then from (34) we conclude that there is a positive probability that

$$
\lim _{i \rightarrow \infty} \hat{H}_{i}=\infty
$$

This implies that $E\left(\lim _{i \rightarrow \infty} \hat{H}_{i}\right)=\infty$, which is inconsistent with the martingale property of $\hat{H}$. Hence $S_{i}$ must converge to an element of $\Sigma$.

Next, we note that this argument can be extended to rule out convergence to any unstable steady state. This is because $\hat{G}$ still contains a negative term of order $1 / i$ when $S_{i}$ is close to the unstable steady state. This term is not summable, which allows us to use the same argument as in the previous paragraph to rule out convergence.

To see this, suppose that we are currently at an unstable steady state; say $S_{i}=S_{k}^{u} \in \Sigma^{u}(Z)$. Then $S_{i+1}$ will be in either of the two basins of attraction above and below this steady state in the next period; let $S_{k+1}^{s}$ be the stable steady state immediately above $S_{k}^{u}$, and let $S_{k}^{s}$ be the stable steady state immediately below $S_{k}^{u}$. In the basin above $S_{k}^{u}, \hat{L}$ has slope $2\left(S_{k}^{u}-S_{k+1}^{s}\right)<0$ (to first order); and in the basin below $S_{k}^{u}, \hat{L}$ has slope $2\left(S_{k}^{u}-S_{k}^{s}\right)>0$.

If $x_{i}=1$, which happens with probability $T_{i}\left(S_{i}, Z\right)$, then $S_{i+1}$ rises by $\left(1-S_{i}\right) /(i+1)$ to $\left(i S_{i}+1\right) /(i+1)$. If $x_{i}=0$, which happens with probability $1-T_{i}\left(S_{i}, Z\right)$, then $S_{i+1}$ falls by $S_{i} /(i+1)$ to $i S_{i} /(i+1)$. Combined with the 
slope information, this means that starting from the unstable steady state $S_{k}^{u}$, $\hat{L}$ is expected to fall by the amount

$$
\hat{G}\left(S_{k}^{u}\right) \approx 2\left[T_{i}\left(S_{i}, Z\right) \frac{1-S_{i}}{i+1}\left(S_{k}^{u}-S_{k+1}^{s}\right)-\left(1-T_{i}\left(S_{i}, Z\right)\right) \frac{S_{i}}{i+1}\left(S_{k}^{u}-S_{k}^{s}\right)\right]
$$

which is negative, and which is not summable with respect to $i$. This calculation can be extended to other points near enough to $S_{k}^{u}$ so that $S_{i+1}^{*} \neq S_{i}^{*}$ with positive probability. Summing these terms, we see that convergence to an unstable steady state with positive probability implies that there is a positive probability that

$$
\lim _{i \rightarrow \infty} \hat{H}_{i}=\infty
$$

which contradicts the martingale property of $\hat{H}$.

QED.

\section{Proof of Proposition 2.}

We fix $Z$, because with full information each $Z$ can be treated separately. Since $u$ is monotone in $\sigma$, policy takes the form of a threshold $\theta(S, Z)$ defined by

$$
u(1, Z, S, \theta)=u(0, Z, S, \theta)
$$

This threshold implies that the fraction playing stock is

$$
\hat{S}=1-F(\theta(S, Z) \mid Z)
$$

For given $Z$, equations (42) and (43) define an increasing function $\hat{S}=T^{f i}(S, Z)$; the set of fixed points $\hat{S}=S$ of this mapping are the full information equilibria associated with the given $Z$.

As the weight $w^{s c} \rightarrow 1$, the utility function approaches $u^{s c}$. In this limit, all individuals play $x=1$ if and only if $S \geq 0.5$, so $T^{f i}$ is vertical at $S=0.5$, and for each $Z, S=0, S=0.5$, and $S=1$ are equilibria.

In the limit as $w^{s c} \rightarrow 0$, the utility function is independent of $S$, so $T^{f i}$ is horizontal, and there is a unique equilibrium for each $Z$.

If we increase heterogeneity in such a way that the density $\partial F / \partial \sigma \rightarrow 0$ at all $\sigma$, then we approach a limit in which every individual either prefers $x=1$, or $x=0$, independently of $S$. Thus $T^{f i}$ is horizontal and equilibrium is unique. Increasing the variance of a normal $F$ is sufficient, but not necessary, for this outcome.

If we contract the distribution to a limit in which all individuals receive the mean signal $\mu_{Z}$, then the $T^{f i}$ function is vertical at the $S^{Z}$ which satisfies $\theta\left(S^{Z}, Z\right)=\mu_{Z}$. If this $S^{Z} \in(0,1)$, then there are three equilibria, $S=0$, $S=S^{Z}$, and $S=1$.

QED. 
To bound the range of full-information equilibria, as we do in the text, note that with our utility function, (42) becomes

$$
w^{\text {info }}(2 Z-1)+w^{s c}(2 S-1)+2 w^{\text {het }} \theta=0
$$

For a normal distribution, approximately $1 \%$ of the probability mass lies on each side of the interval of 2.33 standard deviations around the mean. This means that if the signal is conditionally normal with mean $Z-0.5$ and standard deviation $\eta$, then the highest $Z$ associated with an equilibrium $S$ less than or equal to 0.01 can be calculated from the indifference condition

$$
w^{\text {info }}(2 Z-1)-0.98 w^{s c}+2 w^{\text {het }}(Z-0.5+2.33 \eta)=0
$$

Rearranging, this means that there exists an equilibrium with $S \leq 0.01$ at every $Z$ less than or equal to $0.5+z^{*}$, where $z^{*} \approx\left(0.49 w^{s c}-2.33 w^{\text {het }} \eta\right) /\left(w^{\text {info }}+w^{\text {het }}\right)$.

\section{Proof of Proposition 3.}

We can find equilibria of the global game by searching for threshold signals $\theta^{g g}$ where the individual is indifferent between stock and bond. Such a threshold is a $\theta$ that solves:

$$
\int_{Z \in \mathcal{Z}}[u(1, Z, S(Z), \theta)-u(0, Z, S(Z), \theta)] \frac{f(\theta \mid Z) d \zeta(Z)}{\int_{Z^{\prime} \in \mathcal{Z}} f\left(\theta \mid Z^{\prime}\right) d \zeta\left(Z^{\prime}\right)}=0
$$

where $S(Z)=1-F(\theta \mid Z)$. The integral in the denominator can be ignored since it does not affect the solution for $\theta^{g g}$.

Given our utility function, and the fact that $\sigma$ is distributed $N\left(Z-\frac{1}{2}, \eta\right)$, when $Z$ is 0 or 1 with equal probability this equation becomes:

$$
\sum_{Z \in\{0,1\}}\left[w^{s c}\left(1-2 \Phi\left(\frac{\theta-Z+1 / 2}{\eta}\right)\right)+w^{i n f o}(2 Z-1)+2 w^{\text {het }} \theta\right] \phi\left(\frac{\theta-Z+1 / 2}{\eta}\right)=0
$$

where $\Phi$ and $\phi$ are the standard normal distribution and density.

This equation can be rearranged to give

$$
2 w^{\text {het }} \theta=w^{\text {info }}(1-2 E(Z \mid \theta))+w^{s c}\left[2 E\left(\Phi\left(\frac{\theta-Z+1 / 2}{\eta}\right) \mid \theta\right)-1\right]
$$

In equation (48), the expectation of $Z$ equals the likelihood that $Z=1$, conditional on $\theta$, which we call $l(Z=1 \mid \theta)$ :

$$
E(Z \mid \theta)=l(Z=1 \mid \theta)=\frac{\phi((\theta-1 / 2) / \eta)}{\phi((\theta+1 / 2) / \eta)+\phi((\theta-1 / 2) / \eta)}
$$


In the limit as $\eta \rightarrow 0$, this likelihood becomes a step function which equals 0 for $\theta<0$ and equals 1 for $\theta>0$. Similarly, the expectation of $\Phi$ is

$$
E\left(\Phi\left(\frac{\theta-Z+1 / 2}{\eta}\right) \mid \theta\right)=\Phi\left(\frac{\theta+1 / 2}{\eta}\right) l(Z=0 \mid \theta)+\Phi\left(\frac{\theta-1 / 2}{\eta}\right) l(Z=1 \mid \theta)
$$

where in the limit as $\eta \rightarrow 0, \Phi((\theta+1 / 2) / \eta)$ becomes a step function which is zero below $\theta=-1 / 2$ and one above it; $l(Z=0 \mid \theta)$ is a step function which is one below $\theta=0$ and zero above it; $\Phi((\theta-1 / 2) / \eta)$ is a step function which is zero below $\theta=1 / 2$ and one above it; and $l(Z=1 \mid \theta)$ is as before.

Therefore, using (49) and (50), we see that in the limit as $\eta \rightarrow 0$, the right-hand side of (48) is a step function equal to $-w^{s c}+w^{\text {info }}$ for $\theta<-1 / 2$, $w^{s c}+w^{\text {info }}$ for $-1 / 2<\theta<0,-w^{s c}-w^{\text {info }}$ for $0<\theta<1 / 2$, and $-w^{s c}+w^{\text {info }}$ for $\theta>1 / 2$. Equation (48) has multiple solutions in the limit as $\eta \rightarrow 0$ if and only if $w^{s c} \geq w^{\text {info }}+w^{\text {het }}$.

\section{QED Proposition 3a.}

Next suppose that $\zeta(Z)$ is a normal distribution; assume the same utility function and the same conditional distribution of $\sigma$ as before. Then (46) becomes:

$$
\int_{-\infty}^{\infty}\left[w^{s c}\left(1-2 \Phi\left(\frac{\theta-Z+1 / 2}{\eta}\right)\right)+w^{\text {info }}(2 Z-1)+2 w^{\text {het }} \theta\right] \frac{1}{\eta} \phi\left(\frac{\theta-Z+1 / 2}{\eta}\right) \zeta^{\prime}(Z) d Z=0
$$

We make the change of variables $Y \equiv-Z$ and rearrange to write this as:

$\int_{-\infty}^{\infty}\left[\left(w^{s c}-w^{i n f o}+2 w^{h e t} \theta\right)-2 w^{i n f o} Y-2 w^{s c} \Phi\left(\frac{Y+\theta+1 / 2}{\eta}\right)\right] \frac{1}{\eta} \phi\left(\frac{Y+\theta+1 / 2}{\eta}\right) \zeta^{\prime}(-Y) d Y=0$

The functions $\Phi$ and $\phi$ now refer to a variable $Y$ which has the normal distribution $N(-\theta-1 / 2, \eta)$.

Here we only intend to evaluate this integral in the limit as the standard deviation $\eta$ of $Y$ goes to zero. As we approach this limit, all the weight of the density $\phi$ is concentrated around the mean $-\theta-1 / 2$ of $Y$. While $\Phi$ and $\phi$ are varying rapidly at this point, the other functions of $Y$ that appear in the integral are almost constant. So in the limit, we can treat the expression $2 w^{\text {info }} Y$ as a constant in the integral, equal to $-2 w^{\text {info }}(\theta+1 / 2)$. Likewise $\zeta^{\prime}(-Y) \approx \zeta^{\prime}(\theta+1 / 2)$ near the mean of $Y$, so it can be treated as constant in the integral. Thus in the limit as $\eta \rightarrow 0$, equation (52) becomes:

$$
\int_{-\infty}^{\infty}\left[w^{s c}+2\left(w^{i n f o}+w^{h e t}\right) \theta-2 w^{s c} \Phi\left(\frac{Y+\theta+1 / 2}{\eta}\right)\right] \frac{1}{\eta} \phi\left(\frac{Y+\theta+1 / 2}{\eta}\right) d Y=0
$$


Here, since $\Phi$ is a normal c.d.f., $\int_{-\infty}^{\infty}(1 / \eta) \phi d Y=\int_{-\infty}^{\infty} d \Phi=1$. Likewise, $2 \int_{-\infty}^{\infty} \Phi(1 / \eta) \phi d Y=2 \int_{-\infty}^{\infty} \Phi d \Phi=1$. Therefore, equation (53) becomes

$$
w^{s c}+2\left(w^{i n f o}+w^{h e t}\right) \theta-w^{s c}=0
$$

which is solved only by $\theta^{g g}=0$.

QED Proposition $3 \mathrm{~b}$.

\section{Proof of Proposition 4.}

By Proposition 1, the values of $S$ that occur in equilibrium are distinct, isolated points. We consider only the generic case in which the full-information equilibrium values of $S$ associated with $Z=0$ are distinct from those associated with $Z=1$.

Now since an individual's $n$ observations are a random sample from the whole population, $m / n$ is an unbiassed estimator of $S$. Higher $N^{m a x}$ increases the probability of a large $n$, so for any $\epsilon_{1}, \epsilon_{2}>0, N^{\text {max }}$ can be chosen sufficiently large so that at least fraction $1-\epsilon_{1}$ of the population attributes at least probability $1-\epsilon_{2}$ to the true outcome $(S, Z)$.

Note that the utility function is continuous with respect to all arguments, so that the (possibly multiple) thresholds $\theta_{j}(n, m)$ vary continuously with changes in the inferred probabilities of different outcomes $(S, Z)$. Moreover, we assume that $F$ is continuous, so that a small change in thresholds implies only a small change in the function $T$.

Thus in particular, when there is sufficiently good information about the true state, aggregate behavior is arbitrarily close to what it would be under full information. So suppose we define $T^{N}(S, Z) \equiv T\left(S, Z, y^{*}\right)$ to be the $T$ function in equilibrium when $N^{\max }=N$. Then for any $\epsilon_{3}>0$, and for any outcome $\left(S^{N}, Z\right)$ that occurs with positive probability in herding equilibrium when $N^{\max }=N$, we can choose $N^{\max }$ large enough so that

$$
\left|T^{N}\left(S^{N}, Z\right)-T^{f i}\left(S^{N}, Z\right)\right|<\epsilon_{3}
$$

(Note that this inequality is not satisfied for all $S$, only for those $S^{N}$ which actually occur in herding equilibrium. This is because in herding equilibrium, an individual never attributes positive probability to a non-equilibrium $S$, and therefore $T$ is not close to $T^{f i}$ at such an $S$.)

Thus if $S^{\infty}=\lim _{N \rightarrow \infty} S^{N}$ and $T^{\infty}=\lim _{N \rightarrow \infty} T^{N}$, then we have $T^{\infty}\left(S^{\infty}, Z\right)=$ $T^{f i}\left(S^{\infty}, Z\right)$. The fact that $T^{N}\left(S^{N}, Z\right)$ is on the $45^{\circ}$ line for all $N$ implies that its limit $T^{\infty}\left(S^{\infty}, Z\right)$ is also on the $45^{\circ}$ line.

Thus the equilibria of the limiting herding model are also equilibria of the full information model. So the limiting herding model randomizes over equilibria of the full information model, which is exactly what occurs in a sunspot equilibrium. Of course, this does not rule out the possibility that the limit of the herding model may place all probability on a single full information equilibrium; but it also permits positive probability on more than one full information equilibrium when more than one exists, as occurs in our simulations.

QED. 


\section{Figure 1: Increasing weight on strategic complementarities}
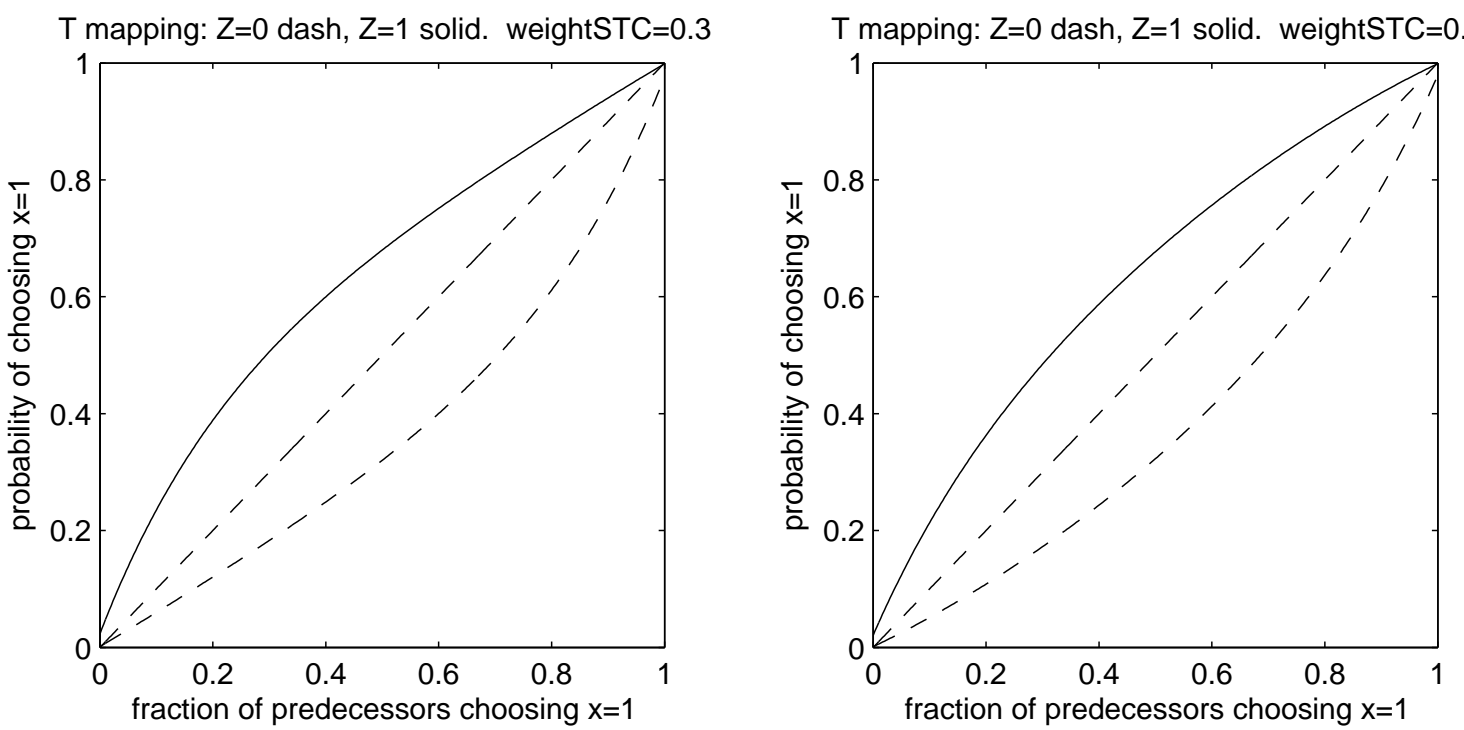

T mapping: $\mathrm{Z}=0$ dash, $\mathrm{Z}=1$ solid. weightSTC $=0.7$
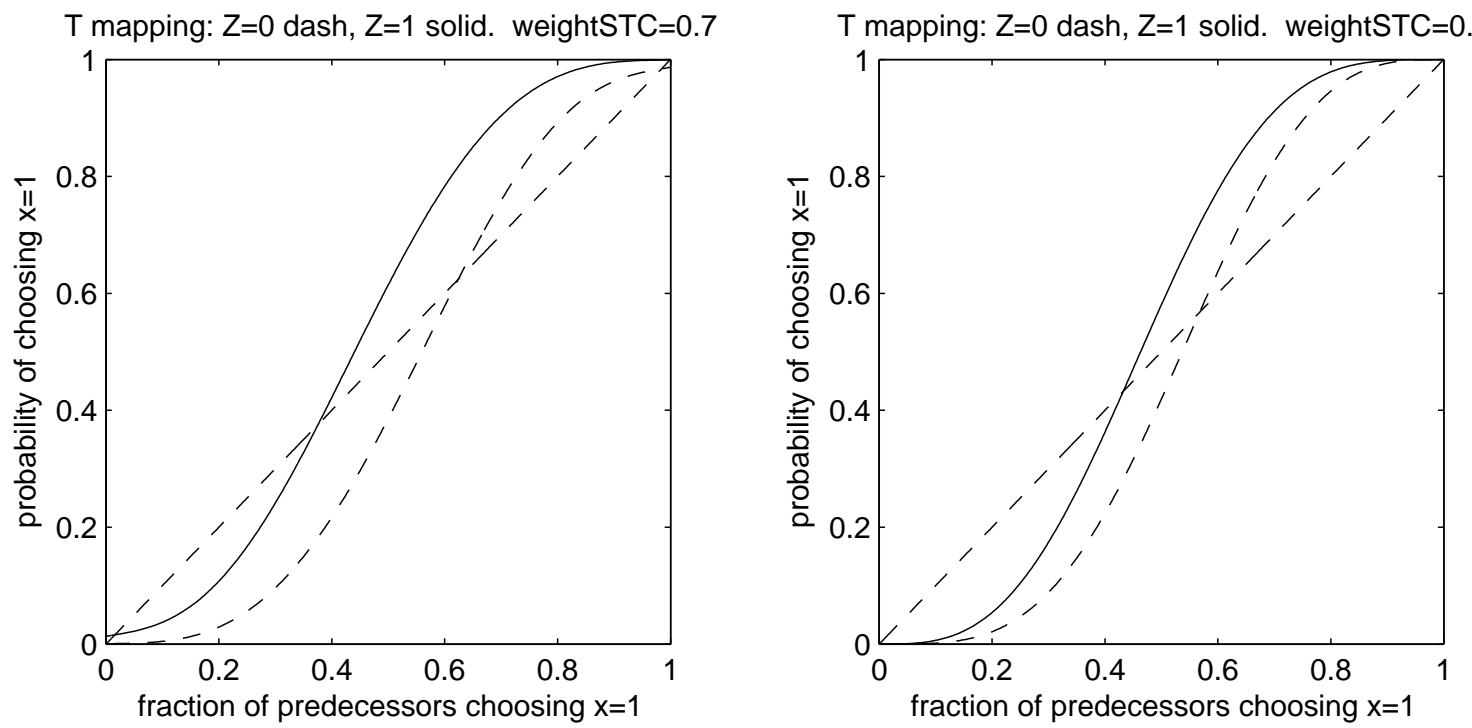
Figure 2: Increasing weight on strategic complementarities
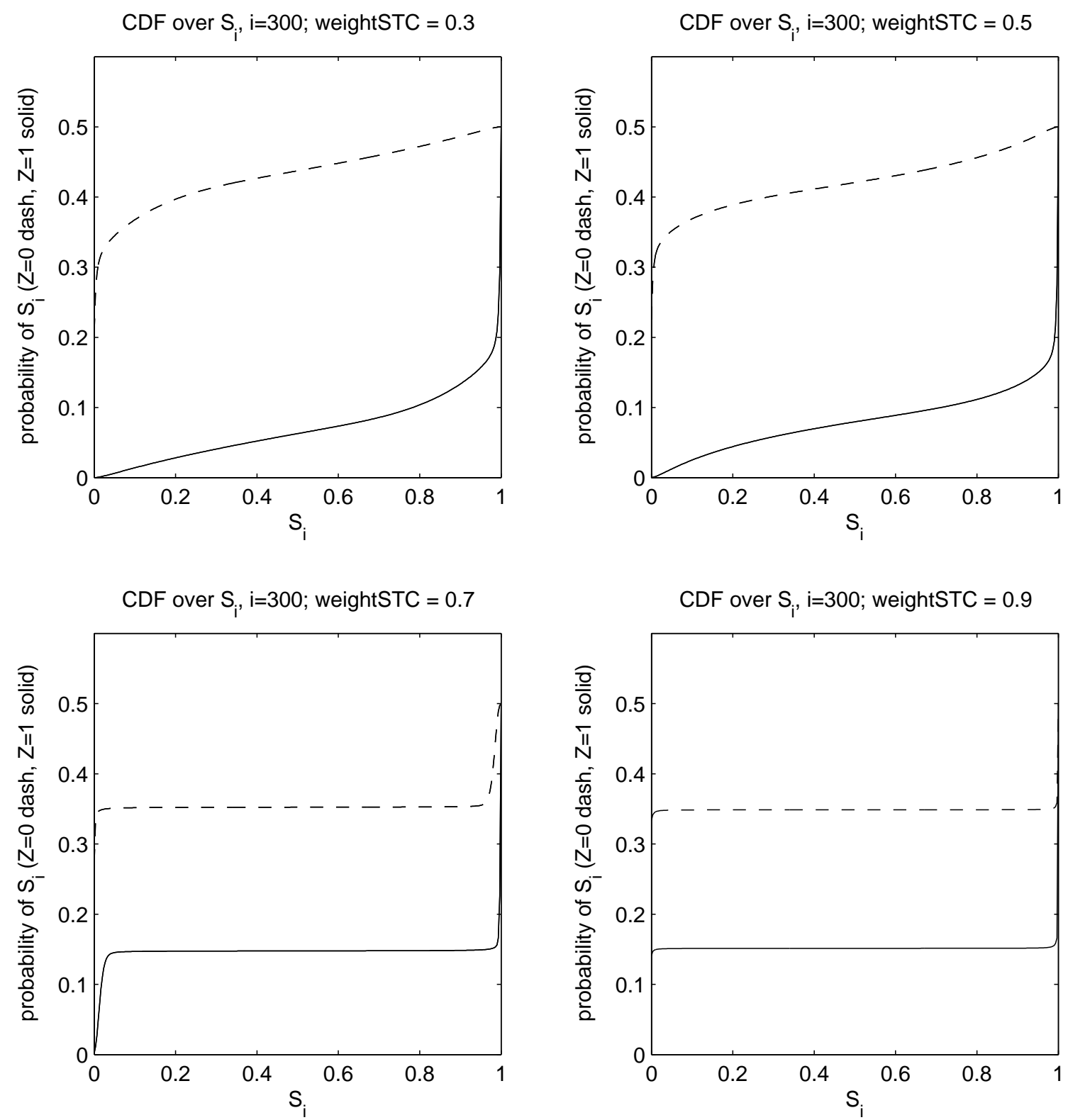
Figure 3: Histories: weak and strong strategic complementarities

Sample of 200 HISTORIES of $S_{i}$ : Agg state $Z=1$, weightSTC $=0.5$

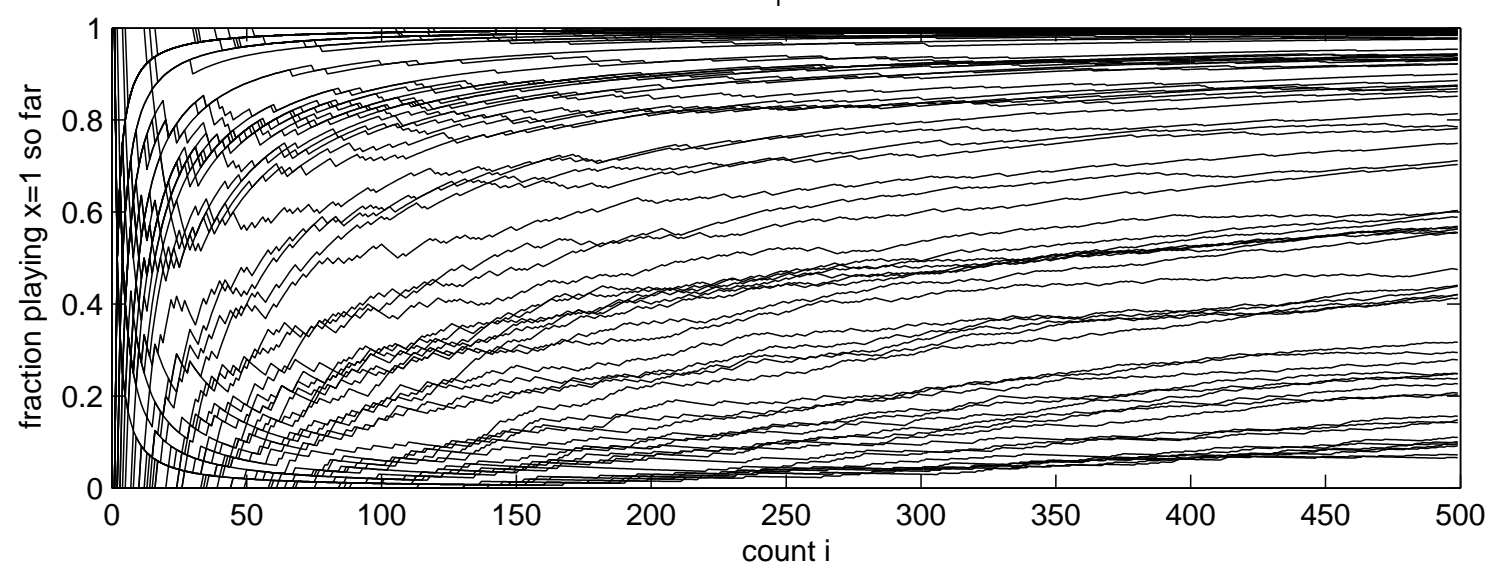

Sample of 200 HISTORIES of $S_{i}$ : Agg state $Z=1$, weightSTC $=0.7$

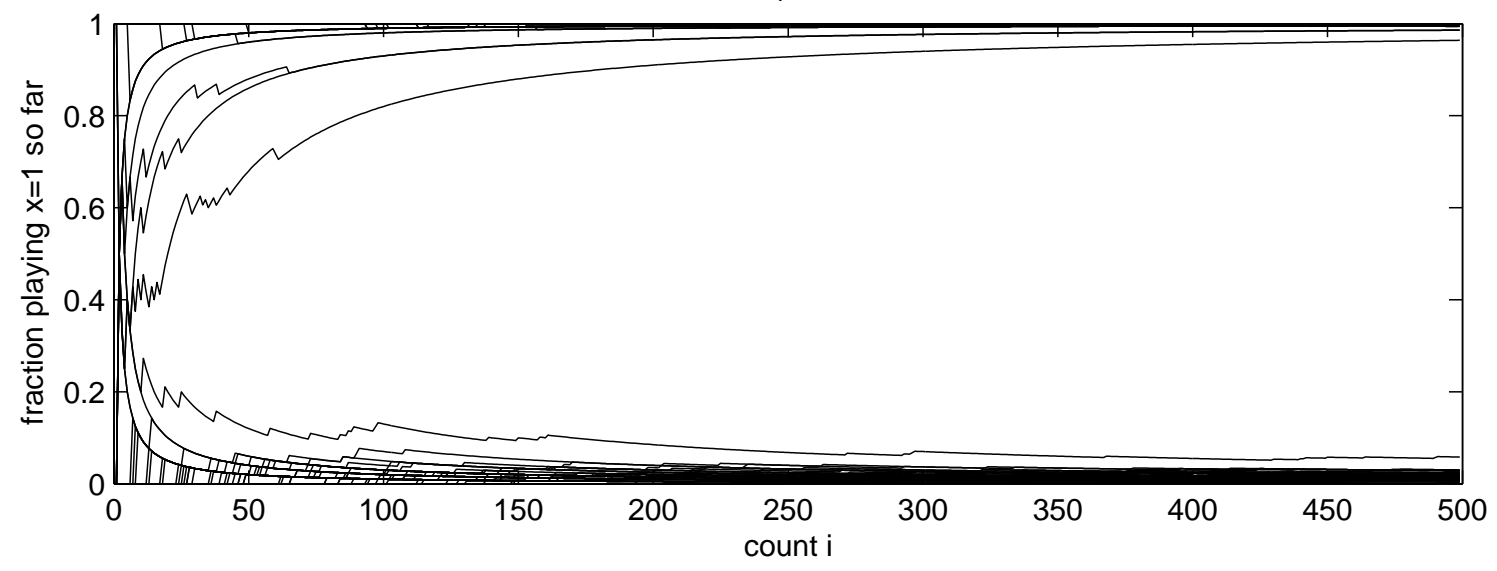

Frequency of $\mathrm{S}_{i}$ : weightSTC $=0.5$
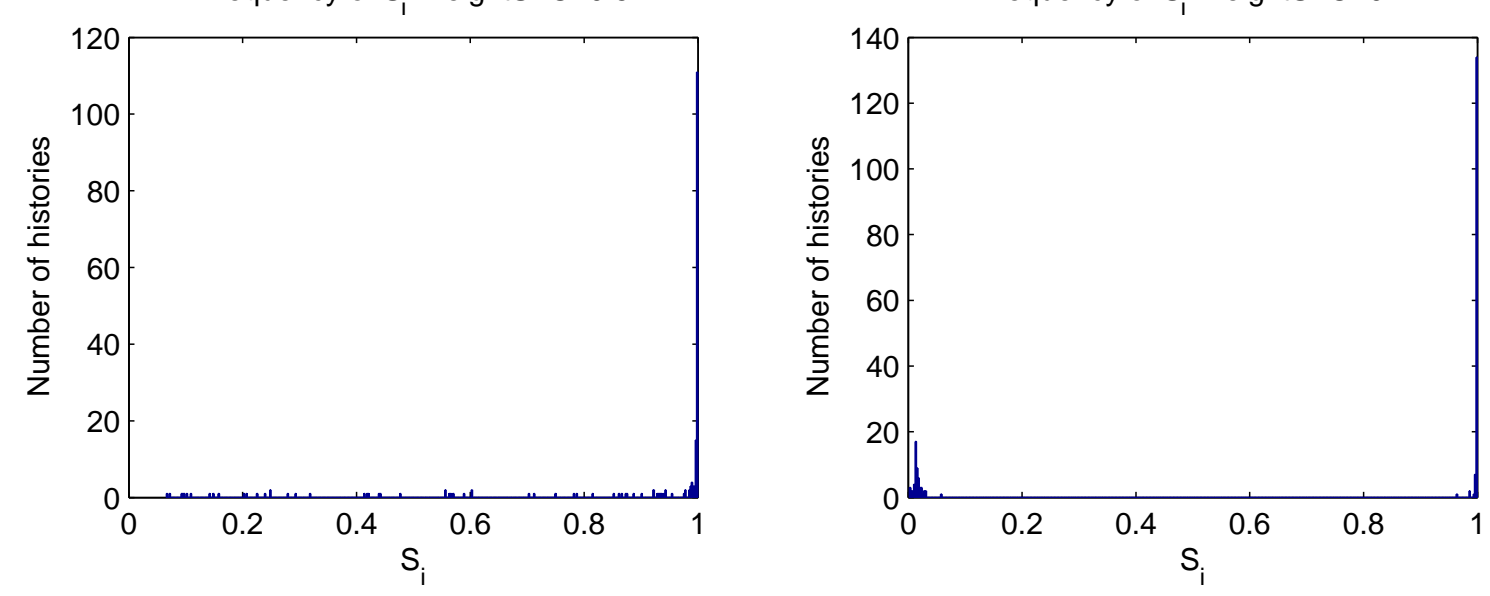
Figure 4: Continuous support for $Z$
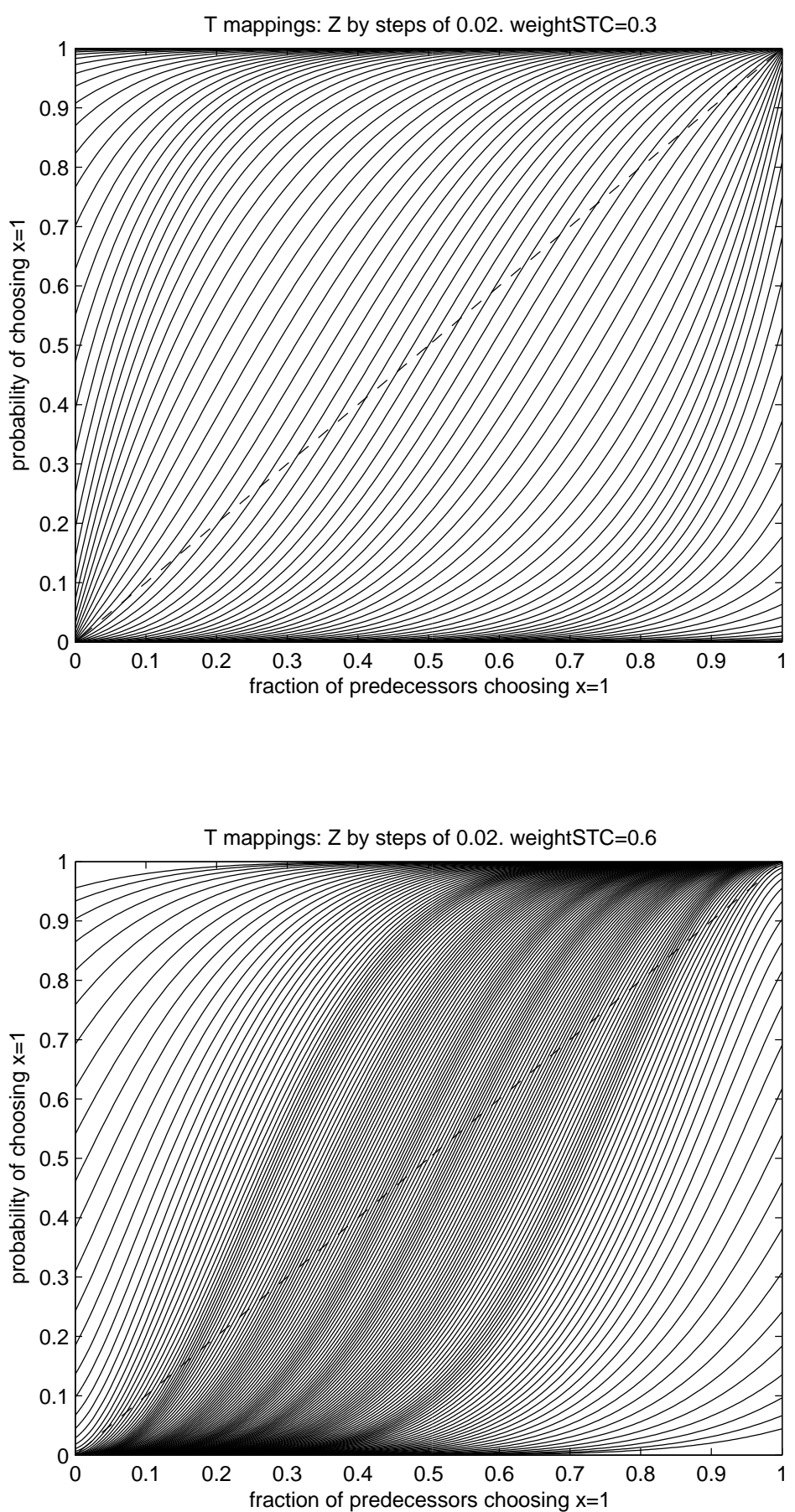


\section{Figure 5: Increasing precision of signal}
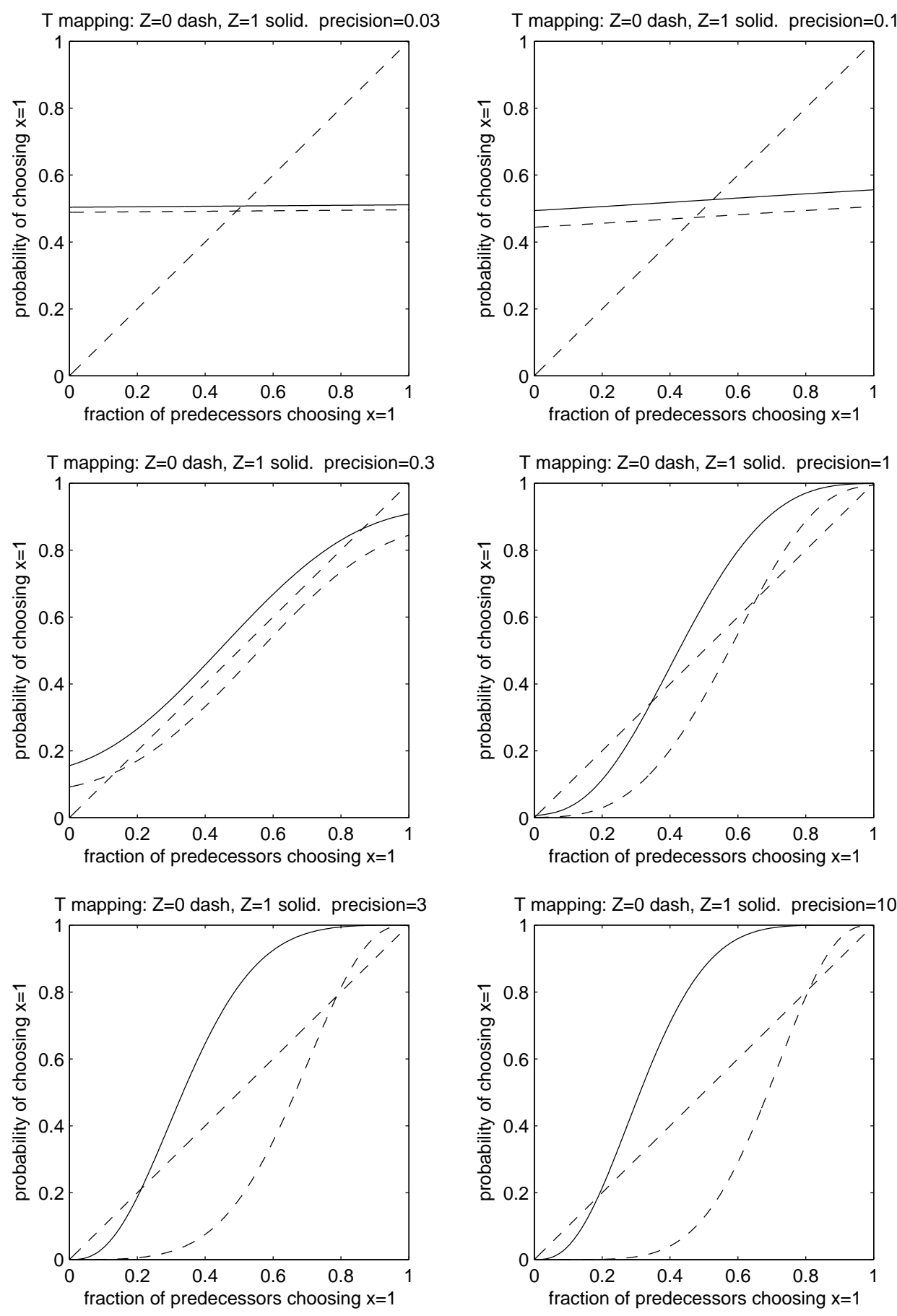


\section{Figure 6: Increasing precision of signal}
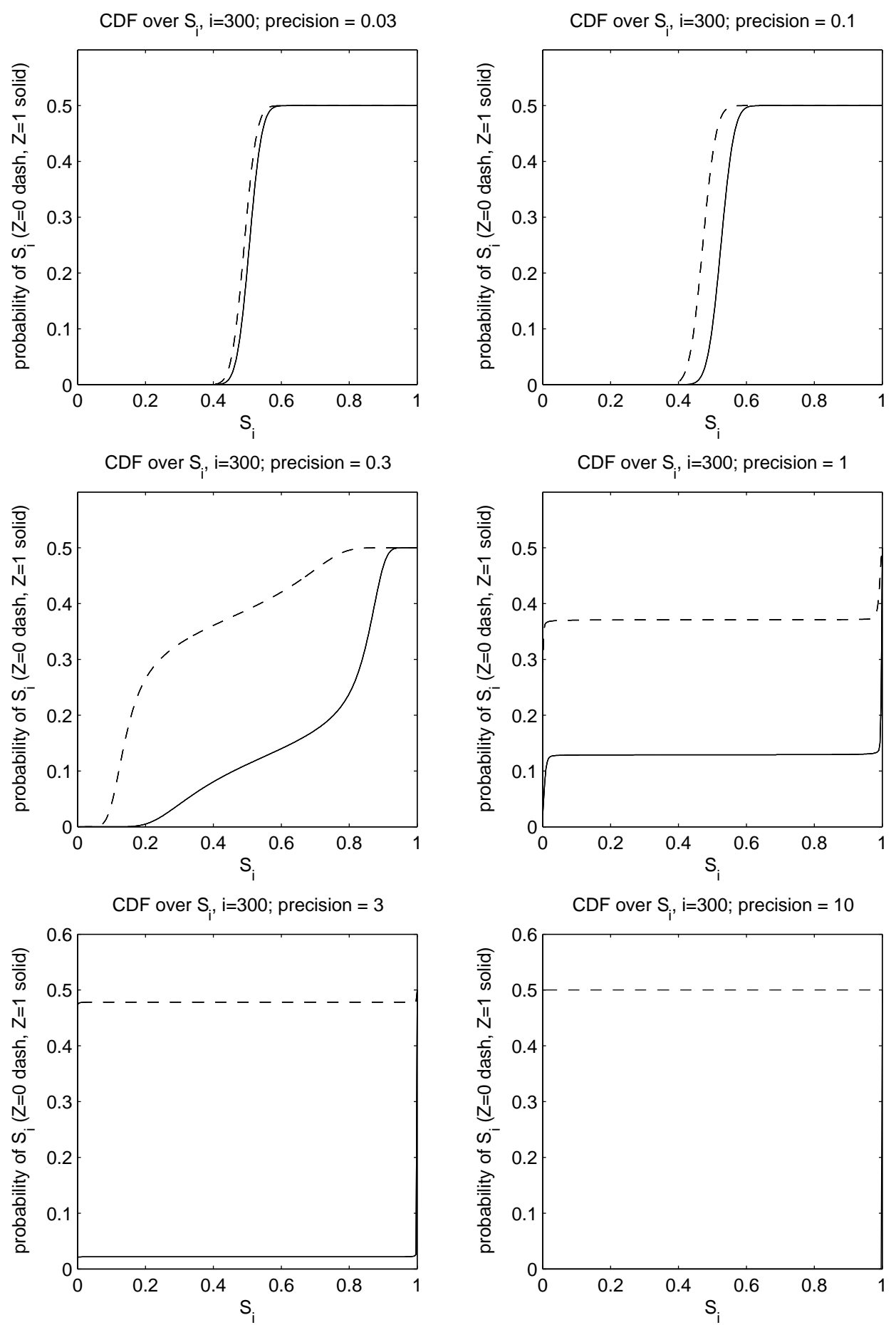


\section{Figure 7: Increasing number observed}
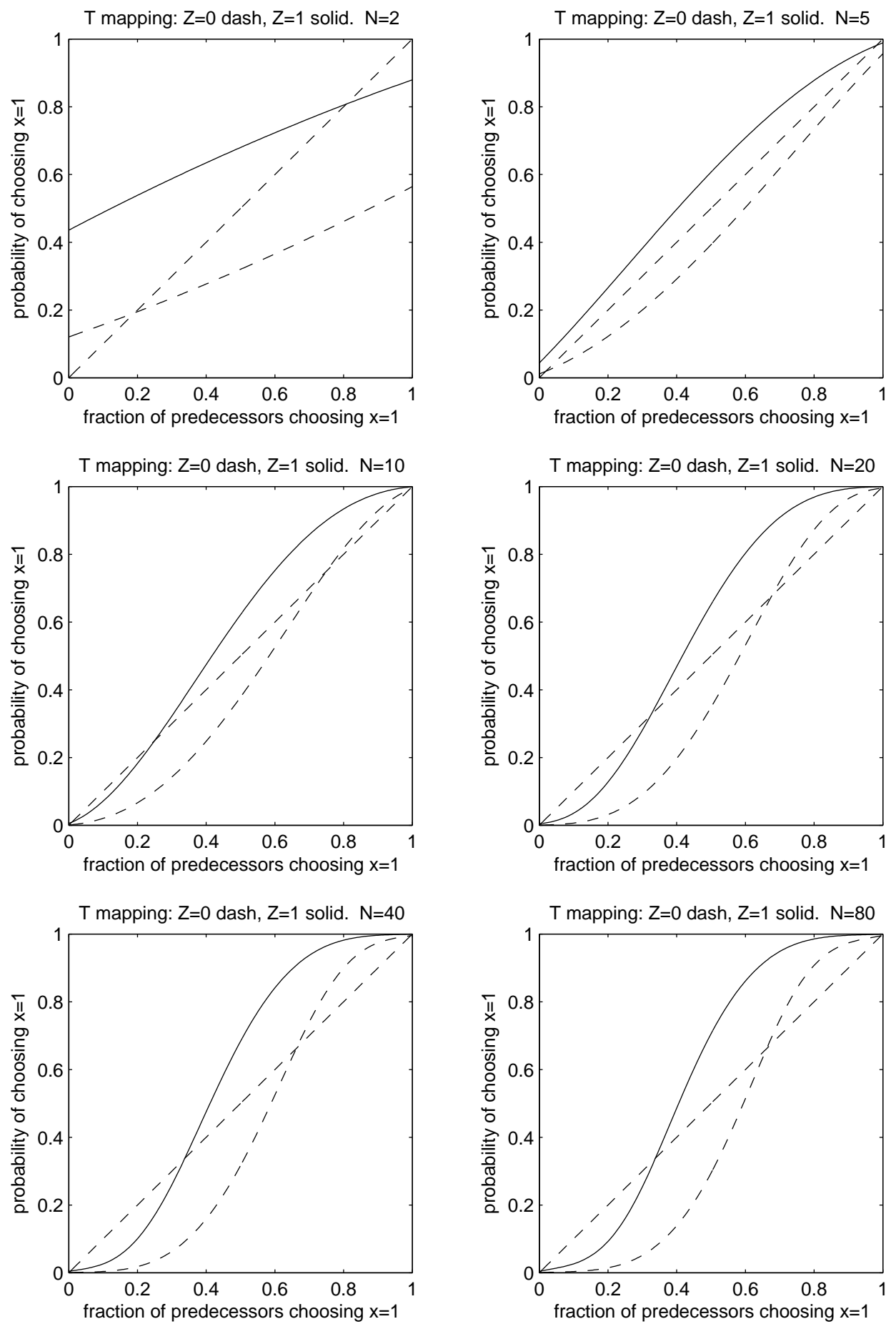


\section{Figure 8: Increasing number observed}
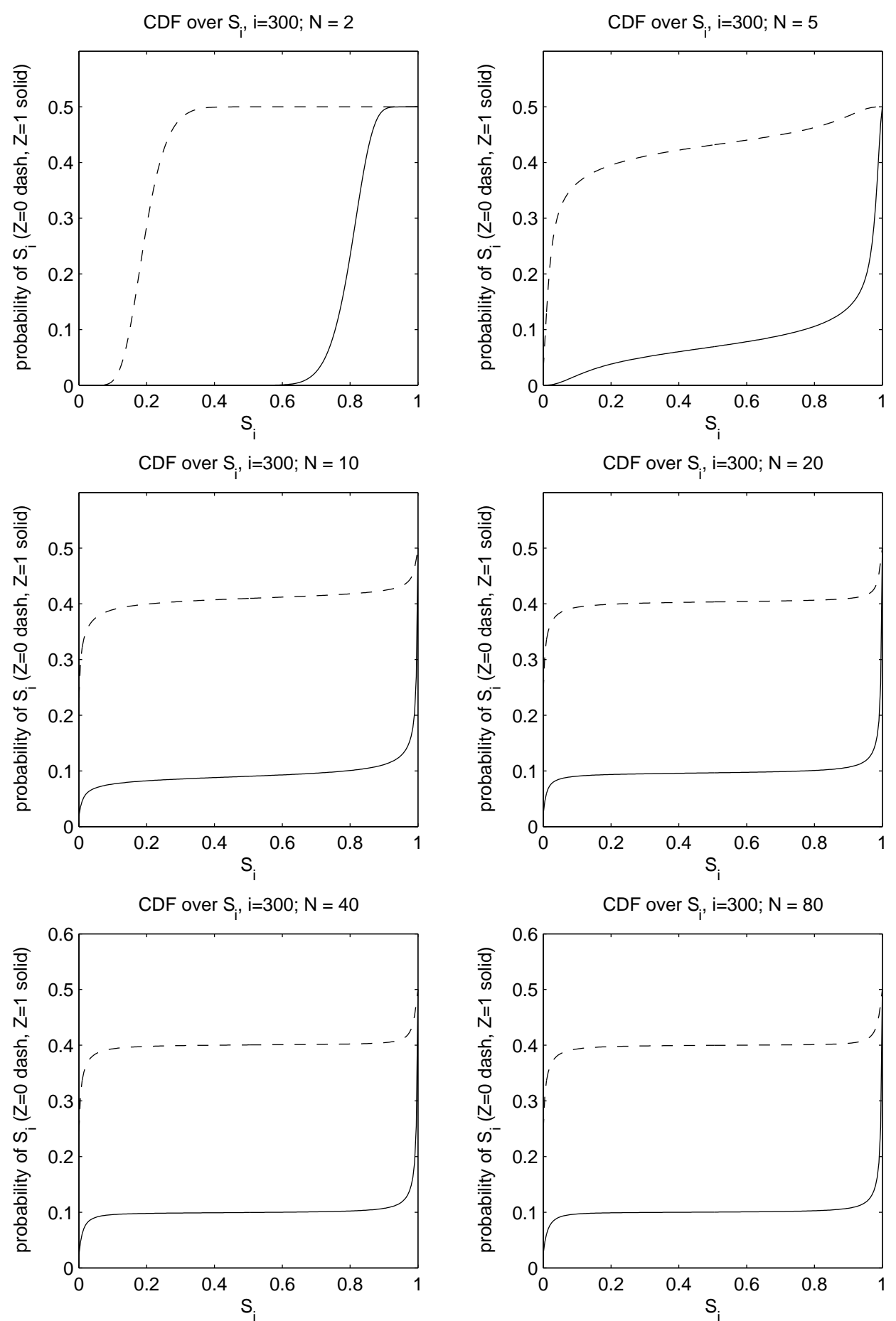